\title{
On the Performance of Free-Space Optical Communication Systems over Double Generalized Gamma Channel
}

\author{
Hessa AlQuwaiee, Student Member, IEEE, Imran Shafique Ansari, Student Member, IEEE, \\ and Mohamed-Slim Alouini, Fellow, IEEE
}

\begin{abstract}
Starting with the double generalized Gamma (GG) model to describe turbulence-induced fading in free-space optical (FSO) systems, we propose a new unified model that accounts for the impact of pointing errors and type of receiver detector. More specifically, we present unified closed-form expressions for the cumulative distribution function, the probability density function, the moment generating function, and the moments of the end-toend signal-to-noise ratio (SNR) of a single link FSO transmission system in terms of the Meijer's G-function. We then use these unified expressions to evaluate performance measures such as the bit error rate, the outage probability, and the ergodic capacity of (i) a single FSO link operating over double GG fading model (ii) asymmetric RF-FSO dual-hop relay transmission system with fixed gain relay. Using an asymptotic expansion of the Meijer's G-function at high SNR, we express all the expressions, derived earlier, in terms of elementary functions. All our analytical results are verified using computer based Monte-Carlo simulations.
\end{abstract}

Index Terms-Free-space optical (FSO) communications, double generalized Gamma, pointing error, heterodyne detection, IM/DD, asymmetric relay system.

\section{INTRODUCTION}

$\mathbf{F}$ REE-space optical (FSO) communication systems can complement and/or substitute radio frequency (RF) systems due to their high bandwidth, robustness to signal interference, and access to license-free spectrum [2], [3]. This makes FSO systems applicable to a variety of broadband wireless technologies such as optical fiber backup, metropolitan area network, enterprise connectivity, and the last mile access networks. However, FSO link performance suffers from the scintillation phenomenon that results from the atmospheric turbulence conditions that can be interpreted as fluctuations of the refractive index caused by inhomogeneous pressure and temperature along with the propagation path. All this leads to varying the laser beam strength at the receiver side that affects the link range and the system reliability [4], [5].

In addition to the scintillation effects, FSO links suffer from thermal expansion, dynamic wind loads, and weak earthquakes that result in vibration of the transmitter beam which

This work was supported by the King Abdulaziz City of Sciences and Technology (KACST) under Grant AT-34-145. Part of this paper was presented at the 4th IEEE International Conference on Communications and Networking (COMNET' 2014), Hammamet, Tunisia, Mar. 2014.

The authors are with Electrical Engineering Program, Computer, Electrical and Mathematical Sciences and Engineering (CEMSE) Division, King Abdullah University of Science and Technology (KAUST), Thuwal 239556900, Kingdom of Saudi Arabia (email: \{hessa.alquwaiee, imran.ansari, slim.alouini\}@kaust.edu.sa). introduces pointing errors due to the misalignment between transmitter and receiver [6], [7]. Moreover, detecting the FSO link beam can be through two techniques, as namely: intensity modulation/direct detection (IM/DD) and heterodyne detection that is more complex than IM/DD but is better in terms of overcoming the turbulence effects [8], [9].

Usually, the irradiance of the atmospheric channels is modeled using the log-normal distribution because of its simplicity but this model only describes weak turbulence conditions. As such, much research work has been reported to propose other statistical models for these type of channels such as the I$\mathrm{K}$ [10], the log-normal Rician [11], Gamma-Gamma [12], [13], double Weibull [14], and extended Generalized-K (EGK) [15] distribution. As a result, Gamma-Gamma model has been widely utilized to analyze FSO systems [6], [7], [16], [17]. In contrast, the study by Chatzidiamantis et al. [14] shows that the double Weibull model is more valid than the GammaGamma model especially for moderate and strong turbulence conditions [18]. Therefore and in a unifying effort, Kashani et al. proposed in [18] a new statistical model for the irradiance called double the generalized Gamma (GG). This new generic proposed model covers all turbulence conditions and is superior over the Gamma-Gamma and double Weibull models based on the numerical results presented in [18, Figs. (1) to (4)].

On another front, improving the performance and enhancing the capacity has been the motive of any newly developed schemes. For instance, relaying technology received over the last decade a great deal of interest as it enhances the capacity of the system in addition to providing a wider coverage. As such, multiple research works have been reported to analyze the relay system for both symmetric and asymmetric links (i.e. symmetric links when source-relay (S R) and relay-destination $(\mathrm{R} \sim \mathrm{D})$ links fall under the same fading model) [19]-[22]. Asymmetric link is more practical and can be expected in a real-life environment as the received signals can be transmitted via different communication systems. For this purpose, in this work, we consider the same relaying system model studied in [17], [23], where multiple RF signals are multiplexed over a single FSO link.

In this work, we first integrate the pointing error model proposed by [24] in the distribution of the irradiance proposed by Kashani et al. in [18]. Moreover, we derive the cumulative distribution function (CDF), the probability density function (PDF), the moment generating function (MGF), and the mo- 
ments in closed-form of the end-to-end signal-to-noise (SNR) of (i) a single FSO link system and (ii) RF-FSO dual-hop transmission system shown in [17, Fig. (1)]. Subsequently, we evaluate some performance metrics such as the average bit error rate (BER) of binary modulation schemes, the outage probability (OP), and the ergodic capacity (EC) of both FSO systems operating over the double GG fading channels. Moreover, all our derivations that are in terms of the Meijer's G-function have been expressed asymptotically for high SNR regime in terms of simple elementary function based on an asymptotic expansion of the Meijer's G-function. This paper is different than any other previously published work such as (i.e. [6], [7], [23], [25]) for some reasons such as the irradiance statistical model that is the double GG is more accurate in modeling the turbulence conditions comparing to the GammaGamma and double Weibull models. Moreover, based on the results of a single link FSO system, we derive the results of the relay assisted link RF/FSO. In addition, our analysis counts for the pointing errors effect and valid for heterodyne and IM/DD detection. Finally, major performance measures are being addressed in exact and asymptotic analysis at high and low SNR regimes.

The rest of the paper is organized as follows. In section II, we present the channel model as well as, exact, and asymptotic expressions of the unified statistics such as the CDF, the PDF, the MGF, and the moments and performance measures such as the OP, the BER, and the EC of a single link FSO system operating over a double GG channel. Next in section III, we study the performance of the RF-FSO dual-hop fixed gain relay transmission system. We list exact closed-form expressions and the asymptotic expressions of the statistics of the end-to-end SNR and evaluate the BER, the OP, and the EC of the system. Finally, we show some numerical examples in Section IV before concluding the paper in Section V.

\section{Single FSO Link SySteM}

\section{A. Channel and System Model}

In this section, we consider a single FSO link with two types of detection techniques, heterodyne and IM/DD. Data transmission is affected by path loss, atmospheric turbulence conditions, pointing errors, and additive white Gaussian noise (AWGN) that can be modeled as

$$
y=\eta I x+w,
$$

where $\eta$ is the effective photoelectric conversion ratio, $w$ refers to the AWGN sample with power spectral density equals to $N_{0}$, and $I$ is the receiver irradiance that is defined as $I=$ $I_{0} I_{a} I_{p}$ where $I_{0}$ is the path loss effect and it is assumed to be normalized to 1 , while $I_{a}$ and $I_{p}$ reflect the turbulence-induced fading and the pointing error effect, respectively [5].

Based on the study of Farid and Hranilovic [24], the pointing loss $I_{p}$ is given by

$$
I_{p}=A_{0} \exp \left(-\frac{2 a^{2}}{w_{e}^{2}}\right)
$$

where $a$ is the radical displacement, $A_{0}$ is the fraction of the collected power when $a=0$ and given by $A_{0}=\operatorname{erf}^{2}(b)$, and $w_{e}$ is the equivalent beam width, defined as $w_{e}=\sqrt{\frac{\sqrt{\pi} \operatorname{erf}(b) w_{b}^{2}}{2 v e^{b^{2}}}}$ such that $w_{b}$ is the beam waist, $b=\sqrt{\frac{\pi}{2}} \frac{R}{w_{b}}, R$ is the aperture radius, and erf(.) is the error function [5], [24]. The pointing error results from vertical and horizontal buildings sway that is independent and identically Gaussian distributed with variance $\sigma_{s}^{2}$, and from radical displacement that is Rayleigh distributed. Consequently, the PDF of $I_{p}$ is given as [5], [24]

$$
f_{p}\left(I_{p}\right)=\frac{\xi^{2}}{A_{0}^{\xi^{2}}} I_{p}^{\xi^{2}-1}, \quad 0 \leq I_{p} \leq A_{0}
$$

where $\xi=\frac{w_{e}}{2 \sigma_{s}^{2}}$ is the ratio between the equivalent beam width at the receiver and the pointing error displacement standard deviation (jitter) [7].

On the other hand, the fading effect is due to the atmospheric turbulence conditions and is modeled by the newly proposed double GG distribution [18]

$$
I_{a}=I_{x} I_{y}
$$

such that $I_{x}$ and $I_{y}$ are independent random processes presenting the large-scale and small-scale fluctuations, respectively. They are each modeled by a generalized Gamma distribution [18], [26] $I_{x} \sim G G\left(\alpha_{1}, \beta_{1}, \Omega_{1}\right)$ and $I_{y} \sim$ $G G\left(\alpha_{2}, \beta_{2}, \Omega_{2}\right)$, where $\beta_{1}$ and $\beta_{2}$ are shaping parameters defining the turbulence-induced fading while $\alpha_{1}, \alpha_{2}, \Omega_{1}, \Omega_{2}$ are identified using the variance of the small and large scale fluctuations from [12, Eq. (18)-(20)] inserted in [18, Eq. (8a), (8b), (9)]. Hence, the PDF of $I_{a}$ can be written as [18, Eq. (4)].

$$
\begin{aligned}
f_{I_{a}}\left(I_{a}\right) & =\frac{\alpha_{2} \lambda \sigma^{\beta_{1}-\frac{1}{2}} \lambda^{\beta_{2}-\frac{1}{2}}(2 \pi)^{1-\frac{\sigma+\lambda}{2}}}{\Gamma\left(\beta_{1}\right) \Gamma\left(\beta_{2}\right) I_{a}} \\
& \times G_{\lambda+\sigma, 0}^{0, \lambda+\sigma}\left[\left(\frac{\Omega_{2}}{I_{a}^{\alpha_{2}}}\right)^{\lambda} \frac{\lambda^{\lambda} \sigma^{\sigma} \Omega_{1}^{\sigma}}{\beta_{1}^{\sigma} \beta_{2}^{\lambda}} \mid \begin{array}{c}
1-\kappa_{0} \\
-
\end{array}\right],
\end{aligned}
$$

where $\kappa_{0}=\Delta\left(\sigma: \beta_{1}\right), \Delta\left(\lambda: \beta_{2}\right), \Gamma($.$) is the Gamma function$ as defined in [27, Eq.(8.310)], $\mathrm{G}_{p, q}^{m, n}[$.$] is the Meijer's G-$ function as defined in [27, Eq.(9.301)], $\lambda$ and $\sigma$ are positive integers such as $\frac{\lambda}{\sigma}=\frac{\alpha_{1}}{\alpha_{2}}$ and $\Delta(x: y) \triangleq \frac{y}{x}, \frac{y+1}{x} \ldots, \frac{y+x-1}{x}$. $\lambda$ and $\sigma$ can be easily identified by first taking some possible values of $\lambda(\sigma)$ then calculate the other parameter using the relation $\sigma=\left\lceil\frac{\lambda \times \alpha_{2}}{\alpha_{1}}\right\rceil\left(\lambda=\left\lceil\frac{\sigma \times \alpha_{1}}{\alpha_{2}}\right\rceil\right)$. After that, we end up with many possibilities of $\lambda$ and $\sigma$ as shown in Table. I. The perfect pair is chosen when their ratio error (i.e error $\left.=\frac{\left|\frac{\alpha_{1}}{\alpha_{2}}-\frac{\lambda}{\sigma}\right|}{\frac{\alpha_{1}}{\alpha_{2}}}\right)$ is the minimum as shaded in Table. I. Moreover, Fig. 1 shows that different values of $\lambda$ and $\sigma$ lead to the same result.

It is very important to note that (5) coincides numerically with EGK PDF given in [15, Eq. (3)] and [28, Eq. (26)]. Also, the Malaga distribution [29] that has been proposed as a unifying model for all turbulence conditions reduces to the double GG by setting the appropriate parameters based on the amount of fading matching. Hence, the joint distribution of $I=I_{a} I_{p}$ is given by [5]-[7]

$$
f_{I}(I)=\int_{I_{p}} f_{I \mid I_{p}}\left(I \mid I_{p}\right) f_{I_{p}}\left(I_{p}\right) d I_{p}, \quad 0 \leq I_{p} \leq A_{0},
$$




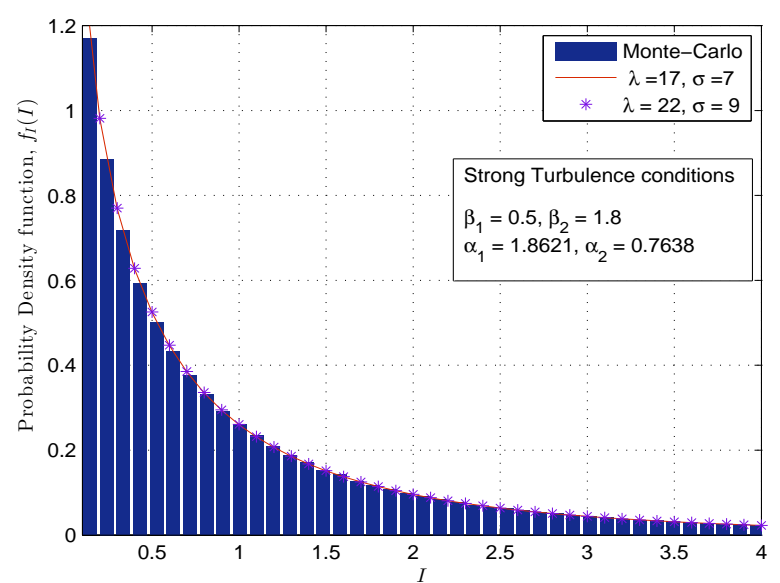

Fig. 1: Comparison between PDFs obtained analytically and via MonteCarlo simulations for different values of $\lambda$ and $\sigma$. TABLE I: Possible values of $\lambda$ and $\sigma$

\begin{tabular}{|c|c|c|c|c|c|}
\hline$\lambda$ & $\sigma$ & Error $\%$ & $\sigma$ & $\lambda$ & Error $\%$ \\
\hline \hline 1 & 1 & 58.98 & 1 & 3 & 23.05 \\
\hline 2 & 1 & 17.96 & 2 & 5 & 2.55 \\
\hline 3 & 2 & 38.47 & 3 & 8 & 9.38 \\
\hline 4 & 2 & 17.96 & 4 & 10 & 2.55 \\
\hline 5 & 3 & 31.63 & 5 & 13 & 6.65 \\
\hline 6 & 3 & 17.96 & 6 & 15 & 2.55 \\
\hline 7 & 3 & 4.29 & 7 & 18 & 5.48 \\
\hline 8 & 4 & 17.96 & 8 & 20 & 2.55 \\
\hline 9 & 4 & 7.70 & 9 & 22 & 0.27 \\
\hline 10 & 5 & 17.96 & 10 & 25 & 2.55 \\
\hline 11 & 5 & 9.76 & 11 & 27 & 0.69 \\
\hline 12 & 5 & 1.56 & 12 & 30 & 2.55 \\
\hline 13 & 6 & 11.13 & 13 & 32 & 0.97 \\
\hline 14 & 6 & 4.21 & 14 & 35 & 2.55 \\
\hline 15 & 7 & 12.10 & 15 & 37 & 1.18 \\
\hline 16 & 7 & 6.24 & 16 & 40 & 2.55 \\
\hline 17 & 7 & 0.38 & 17 & 42 & 1.34 \\
\hline 18 & 8 & 7.71 & 18 & 44 & 0.27 \\
\hline 19 & 8 & 2.59 & 19 & 47 & 1.47 \\
\hline 20 & 9 & 8.85 & 20 & 49 & 0.50 \\
\hline
\end{tabular}

with

$$
f\left(I \mid I_{p}\right)=\frac{1}{I_{p}} f_{I_{a}}\left(\frac{I}{I_{p}}\right), \quad 0 \leq I \leq A_{0} I_{a} .
$$

Inserting (3) and (5) in (6), and using [30, Eq. (07.34.21.0084.01)] yields

$$
\begin{aligned}
f_{I}(I) & =\frac{\xi^{2} \sigma^{\beta_{1}-\frac{1}{2}} \lambda^{\beta_{2}-\frac{1}{2}}(2 \pi)^{1-\frac{\lambda+\sigma}{2}}}{\Gamma\left(\beta_{1}\right) \Gamma\left(\beta_{2}\right) I} \\
& \times \mathrm{G}_{\lambda+\sigma+1,1}^{0, \lambda+\sigma+1}\left[\frac{\lambda^{\lambda} \sigma^{\sigma} \Omega_{1}^{\sigma} \Omega_{2}^{\lambda}}{\beta_{1}^{\sigma} \beta_{2}^{\lambda}}\left(\frac{A_{0}}{I}\right)^{\alpha_{2} \lambda} \mid \begin{array}{c}
\kappa_{1} \\
\kappa_{2}
\end{array}\right],
\end{aligned}
$$

where $\kappa_{1}=1-\frac{\xi^{2}}{\alpha_{2} \lambda}, \Delta\left(\sigma: 1-\beta_{1}\right), \Delta\left(\lambda: 1-\beta_{2}\right)$, and $\kappa_{2}=\frac{-\xi^{2}}{\alpha_{2} \lambda}$. It is important to note that when the pointing error effect approaches zero $^{1},(8)$ coincides mathematically and numerically with $[18, \mathrm{Eq}$. (4)].

\section{B. Unified Statistics}

In this section, we find the statistics of the electrical SNR of single link FSO system over double GG channel under the impact of

\footnotetext{
${ }^{1}$ Non-pointing error case occurs when $\xi \rightarrow \infty$ and $A_{0} \rightarrow 1$.
}

pointing errors for various types of detection techniques.

1) Probability Density Function:

a) Heterodyne Detection: In the case of heterodyne detection [7], the average SNR $\mu_{1}$ is defined as $\mu_{1}=\frac{\eta \mathrm{E}[I]}{N_{0}}$ [7], with $\mathrm{E}[I]$ is defined as

$$
\mathrm{E}[I]=\frac{A_{1} B_{1} A_{0}}{\left(1+\xi^{2}\right) A_{2}^{\frac{1}{\alpha_{2} \lambda}}}=h A_{0},
$$

where $\mathrm{E}[$.$] is the expectation operation with respect to (8),$

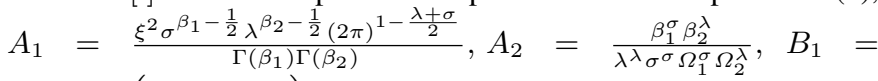
$\prod_{i=1}^{\sigma+\lambda} \Gamma\left(\frac{1}{\alpha_{2} \lambda}+\kappa_{0, i}\right)$, where $\kappa_{u, v}$ is the $v$ th-term of $\kappa_{u}$, and $h=A_{1} B_{1} /\left(1+\xi^{2}\right) A_{2}^{\frac{1}{\alpha_{2} \lambda}}$. So, $\gamma=\frac{n I}{N_{0}}, \frac{\eta}{N_{0}}=\frac{\mu_{1}}{h A_{0}}$, and $I=\frac{\gamma h A_{0}}{\mu_{1}}$. Thus, (8) becomes

$$
f_{\gamma}(\gamma)=\frac{A_{1}}{\gamma} \mathrm{G}_{\lambda+\sigma+1,1}^{0, \lambda+\sigma+1}\left[A_{2}^{-1} h^{-\alpha_{2} \lambda}\left(\frac{\mu_{1}}{\gamma}\right)^{\alpha_{2} \lambda} \mid \begin{array}{c}
\kappa_{1} \\
\kappa_{2}
\end{array}\right] .
$$

b) Intensity Modulation/Direct Detection: Under this type of detection, the average electrical SNR $\mu_{2}$ is given by $\mu_{2}=$ $\frac{(\eta \mathrm{E}[I])^{2}}{N_{0}}$ [5]. Now, with $\gamma=\frac{(\eta I)^{2}}{N_{0}}, \frac{\eta^{2}}{N_{0}}=\frac{\mu_{2}}{h^{2} A_{0}^{2}}$, and $I^{2}=\frac{h^{2} A_{0}^{2} \gamma}{\mu_{2}}$, (8) is simply transformed into

$$
f_{\gamma}(\gamma)=\frac{A_{1}}{2 \gamma} \mathrm{G}_{\lambda+\sigma+1,1}^{0, \lambda+\sigma+1}\left[A_{2}^{-1} h^{-\alpha_{2} \lambda}\left(\frac{\mu_{2}}{\gamma}\right)^{\frac{\alpha_{2} \lambda}{2}} \mid \begin{array}{l}
\kappa_{1} \\
\kappa_{2}
\end{array}\right] .
$$

c) Unified Expression: Observing (10) and (11) and utilizing [30, Eq. (07.34.17.0012.01)], we obtain the following unified PDF

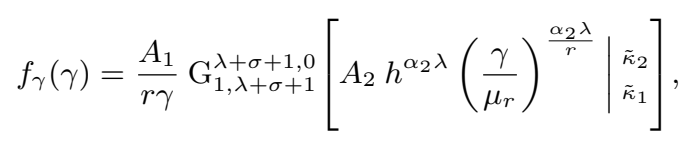

with $^{2}$

$$
\bar{\gamma}_{r}=\frac{\mathrm{E}\left[I^{r}\right]}{\mathrm{E}[I]^{r}} \mu_{r}
$$

where $\tilde{\kappa}_{1}=1-\kappa_{1}=\frac{\xi^{2}}{\alpha_{2} \lambda}, \Delta\left(\sigma: \beta_{1}\right), \Delta\left(\lambda: \beta_{2}\right), \tilde{\kappa}_{2}=1-\kappa_{2}=$ $\frac{\alpha_{2} \lambda+\xi^{2}}{\alpha_{2} \lambda}, \mu_{r}=(\eta \mathbb{E}(I))^{r} / N_{0}, r$ refers to the detection method (i.e. $r=1$ represents heterodyne detection and $r=2$ represents IM/DD). This resulting PDF is generic and it reduces to the Gamma-Gamma fading model with pointing errors case when $\alpha_{1}=\alpha_{2}=1, \Omega_{1}=\Omega_{2}=1, \lambda=\sigma=1, \beta_{1}=\alpha$, and $\beta_{2}=\beta$ as in [13, Eq. (3)] [33, Eq. (1)].

2) Cumulative Distribution Function $(C D F)$ : The CDF defined as $F_{\gamma}(\gamma)=\int_{0}^{\gamma} f_{\gamma}(\gamma) d \gamma$ can be obtained by utilizing [30, Eq. (07.34.21.0084.01)] yielding

$$
\begin{aligned}
F_{\gamma}(\gamma) & =\frac{\xi^{2} \sigma^{\beta_{1}-\frac{1}{2}} \lambda^{\beta_{2}-\frac{1}{2}}(2 \pi)^{1-\frac{r(\lambda+\sigma)}{2}} r^{\beta_{1}+\beta_{2}-2}}{\alpha_{2} \lambda \Gamma\left(\beta_{1}\right) \Gamma\left(\beta_{2}\right)} \\
& \times \mathrm{G}_{r+1, u+1}^{u, 1}\left[C\left(\frac{\gamma}{\mu_{r}}\right)^{v} \mid \begin{array}{c}
1, \kappa_{3} \\
\kappa_{4}, 0
\end{array}\right],
\end{aligned}
$$

where $v=\alpha_{2} \lambda, u=r(\lambda+\sigma+1), C=\left(A_{2} h^{\alpha_{2} \lambda} / r^{\lambda+\sigma}\right)^{r}, \kappa_{3}=$ $\left[\Delta\left(r: \tilde{\kappa}_{2}\right)\right]$ comprising of $r$ terms, and $\kappa_{4}=\left[\Delta\left(r: \tilde{\kappa}_{1}\right)\right]$ comprising of $r(\lambda+\sigma+1)$ terms such that $\left[\Delta\left(z: a_{m}\right)\right] \triangleq$ $\Delta\left(z: a_{1}\right), \Delta\left(z: a_{2}\right) \ldots, \Delta\left(z: a_{m}\right)$. The CDF can be given in a simpler form in terms of basic elementary functions by first

\footnotetext{
${ }^{2}$ The average SNR $\bar{\gamma}$ is defined as $\bar{\gamma}_{r}=\eta^{r} \mathrm{E}\left[I^{r}\right] / N_{0}$ while the average electrical SNR $\mu_{r}$ is given by $\mu_{r}=\eta^{r} \mathbb{E}[I]^{r} / N_{0}$ [31]. Thus, the relation between the average SNR and the average electrical SNR is trivial given that $\frac{\mathrm{E}\left[I^{2}\right]}{\mathrm{E}[I]^{2}} \triangleq \sigma_{s i}^{2}+1$, where $\sigma_{s i}^{2}$ is the scintillation index [32].
} 
inverting the argument of the Meijer's $G$ function using [27, Eq. (9.31.2)] and then applying [13, Eq. (24)]. Thus, the CDF in (14) can be expressed asymptotically at high SNR as

$$
\begin{aligned}
& F_{\gamma}(\gamma) \underset{\mu_{r} \gg 1}{\underset{\sim}{\approx}} \frac{\xi^{2} \sigma^{\beta_{1}-\frac{1}{2}} \lambda^{\beta_{2}-\frac{1}{2}}(2 \pi)^{1-\frac{r(\lambda+\sigma)}{2}} r^{\beta_{1}+\beta_{2}-2}}{\alpha_{2} \lambda \Gamma\left(\beta_{1}\right) \Gamma\left(\beta_{2}\right)} \\
& \times \sum_{k=1}^{u}\left[C^{-1}\left(\frac{\mu_{r}}{\gamma}\right)^{v}\right]^{-\kappa_{4, k}} \frac{\prod_{l=1, l \neq k}^{u} \Gamma\left(\kappa_{4, l}-\kappa_{4, k}\right)}{\kappa_{4, k} \prod_{l=1}^{r} \Gamma\left(\kappa_{3, l}-\kappa_{4, k}\right)} .
\end{aligned}
$$

Hence, the asymptotic expression of the CDF is dominated by $\min \left(\frac{\xi^{2}}{r v}, \frac{\beta_{1}}{r \sigma}, \frac{\beta_{2}}{r \lambda}\right)$ where $\frac{\xi^{2}}{r v}$ represents the first term, $\frac{\beta_{1}}{r \sigma}$ represents the $(r+1)^{t h}$ term and $\frac{\beta_{2}}{r \lambda}$ represents the $(r+r \sigma+1)^{t h}$ term in $\kappa_{4}$ i.e when the difference between the parameters is big enough then the asymptotic expression of the CDF in (15) is dominated by a single term corresponds to the minimum of the three parameters. On the other hand, if the difference between any two parameters is not significance, then the asymptotic expression of the CDF in (15) is dominated by the summation of two terms corresponding to the first and second ranked minimum of the three parameters mentioned above.

3) Moment Generating Function: The MGF defined as $\mathcal{M}_{\gamma}(s) \triangleq \mathbb{E}\left[e^{-\gamma s}\right]$ can be expressed in terms of CDF using integration by parts as [13, Eq. (6)]

$$
\mathcal{M}_{\gamma}(s)=s \int_{0}^{\infty} \exp (-\gamma s) F_{\gamma}(\gamma) \text {. }
$$

Substituting (14) in (16) and utilizing [30, Eq. (07.34.21.0088.01)], the MGF can be expressed as

$$
\begin{aligned}
\mathcal{M}_{\gamma}(s) & =\frac{\xi^{2} \sigma^{\beta_{1}-\frac{1}{2}} \lambda^{\beta_{2}-\frac{1}{2}}(2 \pi)^{\frac{3-r(\lambda+\sigma)-\alpha_{2} \lambda}{2}} r^{\beta_{1}+\beta_{2}-2}}{\sqrt{\alpha_{2} \lambda} \Gamma\left(\beta_{1}\right) \Gamma\left(\beta_{2}\right)} \\
& \times \mathrm{G}_{r+v, u}^{u, v}\left[C\left(\frac{v}{s \mu_{r}}\right)^{v} \mid \begin{array}{c}
\Delta(v: 1), \kappa_{3} \\
\kappa_{4}
\end{array}\right] .
\end{aligned}
$$

In a similar fashion, the MGF can be given asymptotically at high SNR as

$$
\begin{aligned}
& \mathcal{M}_{\gamma}(s) \underset{\mu_{r} \gg 1}{\approx} \frac{\xi^{2} \sigma^{\beta_{1}-\frac{1}{2}} \lambda^{\beta_{2}-\frac{1}{2}}(2 \pi)^{\frac{3-r(\lambda+\sigma)-\alpha_{2} \lambda}{2}} r^{\beta_{1}+\beta_{2}-2}}{\sqrt{\alpha_{2} \lambda} \Gamma\left(\beta_{1}\right) \Gamma\left(\beta_{2}\right)} \\
& \times \sum_{k=1}^{u}\left[C^{-1}\left(\frac{s \mu_{r}}{v}\right)^{v}\right]^{-\kappa_{4, k}} \\
& \times \frac{\prod_{l=1, l \neq k}^{u} \Gamma\left(\kappa_{4, l}-\kappa_{4, k}\right) \prod_{l=1}^{v} \Gamma\left(\{\Delta(v: 0)\}_{l}+\kappa_{4, k}\right)}{\prod_{l=1}^{r} \Gamma\left(\kappa_{3, l}-\kappa_{4, k}\right)} .
\end{aligned}
$$

Again, the dominant terms are similar to the CDF case in Section II-B2.

4) Moments: The moments are defined as

$$
\mathbb{E}\left[\gamma^{n}\right] \triangleq \int_{0}^{\infty} \gamma^{n} f_{\gamma}(\gamma) d \gamma
$$

Placing (12) in (19) and utilizing [27, Eq. (7.813.1)], we obtain the following simple closed-form expression

$$
\mathbb{E}\left[\gamma^{n}\right]=\frac{A_{1} B_{2} \mu_{r}^{n}}{\left(n r+\xi^{2}\right) h^{n r} A_{2}^{\frac{n r}{\alpha_{2} \lambda}}}
$$

where $B_{2}=\prod_{i=1}^{\sigma+\lambda} \Gamma\left(\frac{n r}{v}+\kappa_{0, i}\right)$.

\section{Applications to Performance Analysis}

Based on our results in Section II-B, we now study the performance of a single FSO link operating over double GG fading channel.

1) Outage Probability (OP): The outage probability $P_{\text {out }}$ is defined as follows:

$$
P_{\text {out }}=\operatorname{Pr}\left[\gamma<\gamma_{t h}\right]=F_{\gamma}\left(\gamma_{t h}\right)
$$

Accordingly, the OP for the average SNR is obtained by substituting (14) into (21).

2) Higher-Order Amount of Fading $(A F)$ : Here, we present the amount of fading that can be considered as an important measure to assess the performance of any wireless communication system. The AF aims to define the distribution of the SNR of the received signal [17]. The $n^{t h}$-order AF for $\gamma$ is defined as $A F_{\gamma}=\mathrm{E}\left[\gamma^{n}\right] / \mathrm{E}[\gamma]^{n}-1$ [34]. Using this definition and substituting (20) into it, we obtain the $n^{t h}$-order AF as

$$
A F_{\gamma}^{(n)}=\frac{\left(r+\xi^{2}\right)^{n}}{A_{1}^{n-1} B_{2}^{n-1}\left(n r+\xi^{2}\right)}-1 .
$$

3) Average Bit Error Rate (BER): Using the definition of the average BER [35, Eq. (12)]

$$
\bar{P}_{b}=\frac{q^{p}}{2 \Gamma(p)} \int_{0}^{\infty} \exp (-q \gamma) \gamma^{p-1} F_{\gamma}(\gamma) d \gamma
$$

by placing (14) into (23) and utilizing [30, Eq. (07.34.21.0088.01)], we obtain $\bar{P}_{b}$ as

$$
\begin{aligned}
\bar{P}_{b} & =\frac{\xi^{2} \sigma^{\beta_{1}-\frac{1}{2}} \lambda^{\beta_{2}-\frac{1}{2}}(2 \pi)^{\frac{3-r(\lambda+\sigma)-\alpha_{2} \lambda}{2}} r^{\beta_{1}+\beta_{2}-2}}{2 \Gamma(p) \Gamma\left(\beta_{1}\right) \Gamma\left(\beta_{2}\right)\left(\alpha_{2} \lambda\right)^{\frac{3}{2}-p}} \\
& \times \mathrm{G}_{r+v+1, u+1}^{u, v+1}\left[C\left(\frac{v}{q \mu_{r}}\right)^{v} \mid \begin{array}{c}
\Delta(v: 1-p), 1, \kappa_{3} \\
\kappa_{4}, 0
\end{array}\right],
\end{aligned}
$$

where $p$ and $q$ indicate different binary modulation scheme parameters [17, Table I]. The BER in (24) can be approximated at high SNR using elementary functions as follows

$$
\begin{aligned}
\bar{P}_{b} \underset{\mu_{r} \gg 1}{\underset{\approx}{\approx}} \frac{\xi^{2} \sigma^{\beta_{1}-\frac{1}{2}} \lambda^{\beta_{2}-\frac{1}{2}}(2 \pi)^{\frac{3-r(\lambda+\sigma)-\alpha_{2} \lambda}{2}} r^{\beta_{1}+\beta_{2}-2}}{2 \Gamma(p) \Gamma\left(\beta_{1}\right) \Gamma\left(\beta_{2}\right)\left(\alpha_{2} \lambda\right)^{\frac{3}{2}-p}} \\
\times \sum_{k=1}^{u}\left[C^{-1}\left(\frac{q \mu_{r}}{v}\right)^{v}\right]^{-\kappa_{4, k}} \\
\times \frac{\prod_{l=1, l \neq k}^{u} \Gamma\left(\kappa_{4, l}-\kappa_{4, k}\right) \prod_{l=1}^{v} \Gamma\left(\{\Delta(v: p)\}_{l}+\kappa_{4, k}\right)}{\kappa_{4, k} \prod_{l=1}^{r} \Gamma\left(\kappa_{3, l}-\kappa_{4, k}\right)} .
\end{aligned}
$$

The dominant terms for $\bar{P}_{b}$ are obtained the same way as the $\mathrm{CDF}$ was treated in Section II-B2. If the conditions of a single dominant term are satisfied then (25) can be reduced to the very simple form of [36]

$$
\bar{P}_{b} \approx\left(G_{c} \cdot \mu_{r}\right)^{-G_{d}},
$$

where $G_{c}$ refers to the coding gain while $G_{d}$ is referred to as the diversity gain. Hence the diversity and coding of the system under consideration gain can be simply expressed in terms of elementary functions

$$
G_{d}=v \min \left(\frac{\xi^{2}}{r v}, \frac{\beta_{1}}{r \sigma}, \frac{\beta_{2}}{r \lambda}\right)
$$


and

$$
\begin{aligned}
& G_{c}=\frac{C^{-1 / v} q}{v} \\
& \times\left[\frac{\xi^{2} \sigma^{\beta_{1}-\frac{1}{2}} \lambda^{\beta_{2}-\frac{1}{2}}(2 \pi)^{\frac{3-r(\lambda+\sigma)-\alpha_{2} \lambda}{2}}}{2 \Gamma(p) \Gamma\left(\beta_{1}\right) \Gamma\left(\beta_{2}\right)\left(\alpha_{2} \lambda\right)^{\frac{3}{2}-p}} r^{\beta_{1}+\beta_{2}-2}\right. \\
& \left.\times \frac{\prod_{l=1, l \neq k}^{u} \Gamma\left(\kappa_{4, l}-\kappa_{4, k}\right) \prod_{l=1}^{v} \Gamma\left(\{\Delta(v: p)\}_{l}+\kappa_{4, k}\right)}{\kappa_{4, k} \prod_{l=1}^{r} \Gamma\left(\kappa_{3, l}-\kappa_{4, k}\right)}\right]^{-1 / G_{d}}
\end{aligned}
$$

where $k$ is the index of the $\min \left(\frac{\xi^{2}}{r v}, \frac{\beta_{1}}{r \sigma}, \frac{\beta_{2}}{r \lambda}\right)$ which can be one of the follwoing possibilities $k=1$ or $k=r+1$ or $k=r+r \sigma+1$.

4) Ergodic Capacity: The ergodic capacity is defined as

$$
\begin{aligned}
\bar{C} & \triangleq \mathbb{E}\left[\log _{2}(1+\delta \gamma)\right] \\
& =\int_{0}^{\infty} \log _{2}(1+\delta \gamma) f_{\gamma}(\gamma) d \gamma
\end{aligned}
$$

where $\log _{2}(1+\delta \gamma)$ is expressed as $\frac{\ln (1+\delta \gamma)}{\ln (2)}$ and $\delta=1$ for heterodyne detection $(r=1)$ and $\delta=e / 2 \pi$ for IM/DD $(r=$ 2 ). It is very important to note that the expression in (29) is exact for $r=1$ while it is a lower bound for the case of $r=2$ [37, Eq. (26)] [38, Eq. (7.43)]. $\bar{C}$ is obtained by using (30) along with the identities $\ln (1+x)=\mathrm{G}_{2,2}^{1,2}\left[x \mid \begin{array}{l}1,1 \\ 1,0\end{array}\right]$ and [30, Eq. (07.34.21.0013.01)] yielding

$$
\begin{aligned}
\bar{C} & =\frac{\xi^{2} \sigma^{\beta_{1}-\frac{1}{2}} \lambda^{\beta_{2}-\frac{1}{2}}(2 \pi)^{2-\alpha_{2} \lambda-\frac{r(\lambda+\sigma)}{2}} r^{\beta_{1}+\beta_{2}-2}}{\ln (2) \Gamma\left(\beta_{1}\right) \Gamma\left(\beta_{2}\right) \alpha_{2} \lambda} \\
& \times \mathrm{G}_{r+v+1, u+v+1}^{u+v+1, v}\left[C\left(\frac{1}{\delta \mu_{r}}\right)^{v} \mid \begin{array}{l}
\Delta(v: 0), 1, \kappa_{3} \\
\kappa_{4}, \Delta(v: 0), 0
\end{array}\right],
\end{aligned}
$$

Moreover, $\bar{C}$ can be written asymptotically at high SNR as

$$
\begin{aligned}
\bar{C} & \underset{\mu_{r} \gg 1}{\approx} \frac{\xi^{2} \sigma^{\beta_{1}-\frac{1}{2}} \lambda^{\beta_{2}-\frac{1}{2}}(2 \pi)^{2-\alpha_{2} \lambda-\frac{r(\lambda+\sigma)}{2}} r^{\beta_{1}+\beta_{2}-2}}{\ln (2) \Gamma\left(\beta_{1}\right) \Gamma\left(\beta_{2}\right) \alpha_{2} \lambda} \\
& \times \sum_{k=1}^{u+v+1}\left[C^{-1}\left(\delta \mu_{r}\right)^{v}\right]^{-\kappa_{7, k}} \\
& \times \frac{\prod_{l=1, l \neq k}^{u+v+1} \Gamma\left(\kappa_{7, l}-\kappa_{7, k}\right) \prod_{l=1}^{v} \Gamma\left(\{\Delta(v: 1)\}_{l}+\kappa_{7, k}\right)}{\Gamma\left(1-\kappa_{7, k}\right) \prod_{l=1}^{r} \Gamma\left(\kappa_{3, l}-\kappa_{7, k}\right)},
\end{aligned}
$$

where $\kappa_{7}=\kappa_{4}, \Delta(v: 0), 0$ and can be reduced to the dominant terms slightly different then in the case of II-B2. The asymptotic expression of the capacity in (32) is dominated by the summation of two terms, one corresponds to $\min \left(\frac{\xi^{2}}{r v}, \frac{\beta_{1}}{r \sigma}, \frac{\beta_{2}}{r \lambda}, \frac{\epsilon}{v}\right)$ such that $\frac{\epsilon}{v}$ is the $(u+1)^{t h}$ term in $\kappa_{7}$ where $\epsilon$ is a small error introduced to validate the conditions of [13, Eq. (24)]. The second term corresponds to the $(u+2)^{t h}$ of $\kappa_{7}$.

In another way, at high SNR, the asymptotic results can be derived through the moments as [13, Eq. (20)]

$$
\left.\bar{C} \underset{\mu_{r} \gg 1}{\approx} \frac{\partial}{\partial n} \mathbb{E}\left[\gamma^{n}\right]\right|_{n=0}
$$

Thus, high SNR approximation for the ergodic capacity can be obtained by evaluating the first derivative of the moments
(20) at $n=0$ which is given by

$$
\begin{aligned}
& \frac{\partial}{\partial n} \mathbb{E}\left[\gamma^{n}\right]=\frac{A_{1} B_{2} \mu_{r}^{n}}{\left(n r+\xi^{2}\right) h^{n r} A_{2}^{\frac{n r}{v}}} \\
& \times\left[\log \left(\mu_{r}\right)-r \log (h)-\frac{r \log \left(A_{2}\right)}{v}+\frac{r}{v} B_{3}-\frac{r}{n r+\xi^{2}}\right],
\end{aligned}
$$

where $B_{3}=\sum_{i=1}^{\sigma+\lambda} \psi\left(\frac{n r}{v}+\kappa_{0, i}\right)$, such that $\psi($.$) is the$ digamma function [27, Eq. (8.360.1)]. Evaluating (34) at $n=0$, the ergodic capacity at high SNR can be simply expressed as

$$
\begin{aligned}
& \bar{C} \underset{\mu_{r} \gg 1}{\underset{\sim}{\approx}} \frac{\left.A_{1} B_{2}\right|_{n=0}}{\xi^{2}}\left[\log \left(\delta \mu_{r}\right)-r \log (h)-\frac{r \log \left(A_{2}\right)}{v}\right. \\
& \left.\quad+\left.\frac{r}{v} B_{3}\right|_{n=0}-\frac{r}{\xi^{2}}\right] .
\end{aligned}
$$

In addition, the ergodic capacity at low SNR regime can be approximated by the first moment of the SNR. Placing $n=1$ in the moments (20), the asymptotic ergodic capacity at low SNR can be expressed in terms of elementary functions as

$$
\bar{C} \underset{\mu_{r} \ll 1}{\approx} \frac{A_{1} B_{2} \delta \mu_{r}}{\left(r+\xi^{2}\right) h^{r} A_{2}^{\frac{r}{\alpha_{2} \lambda}}} .
$$

\section{MiXed RF-FSO DUAL-HOP RELAY SySTEM}

\section{A. Channel and System Model}

Here, we consider a dual-hop system similar to the model used in [17] in which a relay ( $R$ ) is placed between the source (S) and the destination (D) assuming that the RF link (S-R) experiences Rayleigh fading whose SNR can be modeled by an exponential distribution with a PDF given as $f_{\gamma_{1}}\left(\gamma_{1}\right)=1 / \bar{\gamma}_{1} \exp \left(-\gamma_{1} / \bar{\gamma}_{1}\right)$ [39], and the FSO link (R-D) is assumed to experience turbulence induced fading with the impact of pointing errors whose SNR PDF is given in (12) with $\gamma$ replaced with $\gamma_{2}$.

\section{B. Statistical Properties}

In this section, we list the statistics of the end-to-end SNR, $\gamma$, of an asymmetric RF-FSO dual-hop fixed gain transmission system defined as

$$
\gamma=\frac{\gamma_{1} \gamma_{2}}{\gamma_{2}+G}
$$

Assuming that $\gamma_{1}$ represents the RF hop (S-R link) while $\gamma_{2}$ represents the FSO hop (R-D link) and $G$ is a fixed relay gain [17], [19], [23].

1) Cumulative Distribution Function (CDF): The CDF of $\gamma$ is defined as [19]

$$
\begin{aligned}
F_{\gamma}(\gamma) & =\operatorname{Pr}\left[\frac{\gamma_{1} \gamma_{2}}{\gamma_{2}+G}<\gamma\right] \\
& =\int_{0}^{\infty} \operatorname{Pr}\left[\frac{\gamma_{1} \gamma_{2}}{\gamma_{2}+G}<\gamma \mid \gamma_{2}\right] f_{\gamma_{2}}\left(\gamma_{2}\right) d \gamma_{2} \\
& =1-\exp \left(-\gamma / \bar{\gamma}_{1}\right) \int_{0}^{\infty} \exp \left(-\gamma G / \bar{\gamma}_{1} \gamma_{2}\right) f_{\gamma_{2}}\left(\gamma_{2}\right) d \gamma_{2}
\end{aligned}
$$


Utilizing [30, Eq. (07.34.03.0228.01)] and [27, Eq. (9.31.2)] to transform $\exp \left(-\gamma G / \bar{\gamma}_{1} \gamma_{2}\right)$ to $\mathrm{G}_{1,0}^{0,1}\left[\frac{\bar{\gamma}_{1} \gamma_{2}}{\gamma G} \mid \begin{array}{c}1 \\ -\end{array}\right]$ and then applying it on [30, Eq. (07.34.21.0013.01)], the CDF of $\gamma$ can be expressed as

$$
\begin{aligned}
F_{\gamma}(\gamma) & =1-A_{3} \exp \left(-\gamma / \bar{\gamma}_{1}\right) \\
& \times \mathrm{G}_{r, u+v}^{u+v, 0}\left[C\left(\frac{\gamma G}{v \mu_{2, r} \bar{\gamma}_{1}}\right)^{v} \mid \begin{array}{c}
\kappa_{3} \\
\kappa_{4}, \Delta(v: 0)
\end{array}\right],
\end{aligned}
$$

where $A_{3}=\frac{\xi^{2} \sigma^{\beta_{1}-1 / 2} \lambda^{\beta_{2}-1 / 2}(2 \pi) \frac{3-r(\lambda+\sigma)-\alpha_{2} \lambda}{2} r^{\beta_{1}+\beta_{2}-2}}{\sqrt{\alpha_{2} \lambda} \Gamma\left(\beta_{1}\right) \Gamma\left(\beta_{2}\right)}$. The $\mathrm{CDF}$ can be expressed at high SNR as

$$
\begin{aligned}
& F_{\gamma}(\gamma) \underset{\mu_{r} \gg 1}{\approx} 1-A_{3} \exp \left(-\gamma / \bar{\gamma}_{1}\right) \\
& \times \sum_{k=1}^{u+v}\left[C^{-1}\left(\frac{v \mu_{2, r} \bar{\gamma}_{1}}{\gamma G}\right)^{v}\right]^{-\kappa_{8, k}} \frac{\prod_{l=1, l \neq k}^{u+v} \Gamma\left(\kappa_{8, l}-\kappa_{8, k}\right)}{\prod_{l=1}^{r} \Gamma\left(\kappa_{3, l}-\kappa_{8, k}\right)},
\end{aligned}
$$

where $\kappa_{8}=\kappa_{4}, \Delta(v: 0)$. In the case of the relay link, the dominant terms for (40) and for the asymptotic expressions derived ahead are dominated by the summation of two terms. The first term corresponds to $\min \left(\frac{\xi^{2}}{r v}, \frac{\beta_{1}}{r \sigma}, \frac{\beta_{2}}{r \lambda}, \frac{\epsilon}{v}\right)$ while the second term corresponds to $\frac{\epsilon+v-1}{v}$ that is the $(u+v)^{t h}$ term in $\kappa_{8}$.

2) Probability Density Function (PDF): The PDF is obtained by differentiating (39) with respect to $\gamma$, using the product rule, and then utilizing [30, Eq. (07.34.20.0002.01)] as

$$
\begin{aligned}
& f_{\gamma}(\gamma)=\exp \left(-\frac{\gamma}{\bar{\gamma}_{1}}\right)\left(\frac{A_{3}}{\bar{\gamma}_{1}} \mathrm{G}_{r, u+v}^{u+v, 0}\left[C\left(\frac{\gamma G}{v \mu_{2, r} \bar{\gamma}_{1}}\right)^{v} \mid \begin{array}{c}
\kappa_{3} \\
\kappa_{4}, \Delta(v: 0)
\end{array}\right]\right. \\
& \left.-\frac{A_{3} v}{\gamma} \mathrm{G}_{r+1, m+n+1}^{u+v, 1}\left[C\left(\frac{\gamma G}{v \mu_{2, r} \bar{\gamma}_{1}}\right)^{v} \mid \begin{array}{c}
0, \kappa_{3} \\
\kappa_{4}, \Delta(v: 0), 1
\end{array}\right]\right) \text {. }
\end{aligned}
$$

3) Moment Generating Function (MGF): Substituting (39) in (16) and utilizing [30, Eq. (07.34.21.0088.01)], the MGF can be expressed as

$$
\begin{aligned}
\mathcal{M}_{\gamma}(s) & =1-\frac{s \xi^{2} \sigma^{\beta_{1}-1 / 2} \lambda^{\beta_{2}-1 / 2}(2 \pi)^{\frac{4-r(\lambda+\sigma)-2 \alpha_{2} \lambda}{2}} r^{\beta_{1}+\beta_{2}-2}}{\left(1 / \bar{\gamma}_{1}+s\right) \Gamma\left(\beta_{1}\right) \Gamma\left(\beta_{2}\right)} \\
& \times \mathrm{G}_{r+v, u+v}^{u+v, v}\left[C\left(\frac{G}{\left(1 / \bar{\gamma}_{1}+s\right) \mu_{2, r} \bar{\gamma}_{1}}\right)^{v} \mid \begin{array}{c}
\Delta(v: 0), \kappa_{3} \\
\kappa_{4}, \Delta(v: 0)
\end{array}\right] .
\end{aligned}
$$

Using the asymptotic expansion of the Meijer's G-function, the MGF can be approximated at high SNR with

$$
\begin{aligned}
& \mathcal{M}_{\gamma}(s) \underset{\mu_{r} \gg 1}{\approx} 1-\frac{\sigma^{\beta_{1}-1 / 2} \lambda^{\beta_{2}-1 / 2}(2 \pi)^{\frac{4-r(\lambda+\sigma)-2 \alpha_{2} \lambda}{2}}}{\xi^{-2} s^{-1}\left(1 / \bar{\gamma}_{1}+s\right) \Gamma\left(\beta_{1}\right) \Gamma\left(\beta_{2}\right)} r^{\beta_{1}+\beta_{2}-2} \\
& \times \sum_{k=1}^{u+v}\left[C^{-1}\left(\frac{\left(1 / \bar{\gamma}_{1}+s\right) \mu_{2, r} \bar{\gamma}_{1}}{G}\right)^{v}\right]^{-\kappa_{8, k}} \\
& \times \frac{\prod_{l=1, l \neq k}^{u+v} \Gamma\left(\kappa_{8, l}-\kappa_{8, k}\right) \prod_{l=1}^{v} \Gamma\left(\{\Delta(v: 1)\}_{l}+\kappa_{8, k}\right)}{\prod_{l=1}^{r} \Gamma\left(\kappa_{3, l}-\kappa_{8, k}\right)} .
\end{aligned}
$$

4) Moments: The moments is defined as (19) can be expressed in terms of the complementary $\operatorname{CDF} F_{\gamma}^{c}(\gamma)=$
$1-F_{\gamma}(\gamma)$ as

$$
\mathbb{E}\left[\gamma^{n}\right] \triangleq n \int_{0}^{\infty} \gamma^{n-1} F_{\gamma}^{c}(\gamma) d \gamma .
$$

By using this definition (44) and placing the complementary of (39) into it, we obtain the moments after utilizing [30, Eq. $(07.34 .21 .0088 .01)]$ as

$$
\begin{aligned}
\mathbb{E}\left[\gamma^{n}\right] & =\frac{\bar{\gamma}_{1}^{n} n \xi^{2} \sigma^{\beta_{1}-1 / 2} \lambda^{\beta_{2}-1 / 2}(2 \pi)^{\frac{4-r(\lambda+\sigma)-2 \alpha_{2} \lambda}{2}}}{\left(\alpha_{2} \lambda\right)^{1-n} \Gamma\left(\beta_{1}\right) \Gamma\left(\beta_{2}\right)} r^{\beta_{1}+\beta_{2}-2} \\
& \times \mathrm{G}_{r+v, u+v}^{u+v, v}\left[C\left(\frac{G}{\mu_{2, r}}\right)^{v} \mid \begin{array}{c}
\Delta(v: 1-n), \kappa_{3} \\
\kappa_{4}, \Delta(v: 0)
\end{array}\right]
\end{aligned}
$$

Using again the asymptotic expansion of the Meijer's function, we can express (45) asymptotically at high SNR as

$$
\begin{aligned}
\mathbb{E}\left[\gamma^{n}\right] & \underset{\mu_{r} \gg 1}{\approx} \frac{\bar{\gamma}_{1}^{n} n \xi^{2} \sigma^{\beta_{1}-1 / 2} \lambda^{\beta_{2}-1 / 2}(2 \pi)^{\frac{4-r(\lambda+\sigma)-2 \alpha_{2} \lambda}{2}}}{\left(\alpha_{2} \lambda\right)^{1-n} \Gamma\left(\beta_{1}\right) \Gamma\left(\beta_{2}\right)} r^{\beta_{1}+\beta_{2}-2} \\
& \times \sum_{k=1}^{u+v}\left[C^{-1}\left(\frac{\mu_{2, r}}{G}\right)^{v}\right]^{-\kappa_{8, k}} \\
& \times \frac{\prod_{l=1, l \neq k}^{u+v} \Gamma\left(\kappa_{8, l}-\kappa_{8, k}\right) \prod_{l=1}^{v} \Gamma\left(\{\Delta(v: n)\}_{l}+\kappa_{8, k}\right)}{\prod_{l=1}^{r} \Gamma\left(\kappa_{3, l}-\kappa_{8, k}\right)} .
\end{aligned}
$$

\section{Applications to Performance Analysis}

In this section, we evaluate the performance measures of the dual-hop RF-FSO relay transmission system.

1) Outage Probability $(O P)$ : The outage probability for the SNR is obtained by substituting (39) into (21).

2) Average Bit Error Rate (BER): Placing (39) into (23) and utilizing [30, Eq. (07.34.21.0088.01)], we obtain $\bar{P}_{b}$ as

$$
\begin{aligned}
\bar{P}_{b} & =\frac{1}{2}-\frac{q^{p} \xi^{2} \sigma^{\beta_{1}-1 / 2} \lambda^{\beta_{2}-1 / 2}(2 \pi)^{\frac{4-r(\lambda+\sigma)-2 \alpha_{2} \lambda}{2}}}{2\left(\alpha_{2} \lambda\right)^{1-p}\left(1 / \bar{\gamma}_{1}+q\right)^{p} \Gamma\left(\beta_{1}\right) \Gamma\left(\beta_{2}\right) \Gamma(p)} r^{\beta_{1}+\beta_{2}-2} \\
& \times \mathrm{G}_{r+v, u+v}^{u+v, v}\left[C\left(\frac{G}{\left(1 / \bar{\gamma}_{1}+q\right) \mu_{2, r} \bar{\gamma}_{1}}\right)^{v} \mid \begin{array}{c}
\Delta(v: 1-p), \kappa_{3} \\
\kappa_{4}, \Delta(v: 0)
\end{array}\right] .
\end{aligned}
$$

$\bar{P}_{b}$ can be expressed at high SNR as

$$
\begin{aligned}
\bar{P}_{b} & \underset{\mu_{r} \gg 1}{\approx} \frac{1}{2}-\frac{q^{p} \xi^{2} \sigma^{\beta_{1}-1 / 2} \lambda^{\beta_{2}-1 / 2}(2 \pi)^{\frac{4-r(\lambda+\sigma)-2 \alpha_{2} \lambda}{2}}}{2\left(\alpha_{2} \lambda\right)^{1-p}\left(1 / \bar{\gamma}_{1}+q\right)^{p} \Gamma\left(\beta_{1}\right) \Gamma\left(\beta_{2}\right) \Gamma(p)} r^{\beta_{1}+\beta_{2}-2} \\
& \times \sum_{k=1}^{u+v}\left[C^{-1}\left(\frac{\left(1 / \bar{\gamma}_{1}+q\right) \mu_{2, r} \bar{\gamma}_{1}}{G}\right)^{v}\right]^{-\kappa_{8, k}} \\
& \times \frac{\prod_{l=1, l \neq k}^{u+v} \Gamma\left(\kappa_{8, l}-\kappa_{8, k}\right) \prod_{l=1}^{v} \Gamma\left(\{\Delta(v: p)\}_{l}+\kappa_{8, k}\right)}{\prod_{l=1}^{r} \Gamma\left(\kappa_{3, l}-\kappa_{8, k}\right)}
\end{aligned}
$$

3) Ergodic Capacity: The ergodic capacity in (29) can be expressed in terms of the complementary CDF [40, Eq. (15)] as

$$
\bar{C}=\int_{0}^{\infty} \frac{1}{\ln (2)}(1+\delta \gamma)^{-1} F_{\gamma}^{c}(\gamma) d \gamma .
$$

We utilize the identities [30, Eq. (07.34.03.0271.01), Eq. (07.34.03.0228.01)] to express $(1+\delta \gamma)^{-1}$ and $\exp \left(\gamma / \bar{\gamma}_{1}\right)$ in terms of the Meijer's G-function, respectively. Next, to solve the integral with the product of three G-functions, we transformed each term into the Fox H-function [41] using [30, Eq. (07.34.26.0008.01)] and then we use the identity [42, Eq. (2.3)] to obtain $\bar{C}$ in terms of the H-function of two 
variables [42, Eq. (1.1)]

$$
\begin{aligned}
& \bar{C}=\frac{A_{3} \delta \bar{\gamma}_{1}}{\ln (2) v} \\
& \times \mathrm{H}_{1,0: 1,1: r, u+v}^{0,1: 1,1: u+v, 0}\left[\begin{array}{c|c|c|c}
(0 ; 1,1) & (0,1) & \left(\kappa_{3},\left[(v)^{-1}\right]_{r}\right) & \delta \bar{\gamma}_{1}, \frac{G C^{1 / v}}{v \mu_{2, r}}
\end{array}\right],
\end{aligned}
$$

where $[x]_{i}=x, x, \ldots x$, comprising of $i$ terms. The bivariate $\mathrm{H}$-function can be evaluated efficiently using MATHEMATICA ${ }^{\circledR}$ implementation in [35] or MATLAB ${ }^{\circledR}$ implementation in [43]. In this work, we have implemented the bivariate $\mathrm{H}$-function in MATHEMATICA ${ }^{\circledR}$ and easily evaluated the expression in (50).

In addition, we can obtain an asymptotic expression of the ergodic capacity utilizing the expansion of the Meijer's Gfunction. In other words, exploiting the asymptotic expression of the CDF in (40) to be then inserted in (49) leading to

$$
\begin{aligned}
\bar{C} \underset{\mu_{r} \gg 1}{\approx} \frac{A_{3}}{\ln (2)} \exp \left(1 / \delta \bar{\gamma}_{1}\right) \\
\quad \times \sum_{k=1}^{u+v} C^{\kappa_{8, k}}\left(\frac{G}{v \delta \bar{\gamma}_{1} \mu_{2, r}}\right)^{v \kappa_{8, k}} \Gamma\left(-v \kappa_{8, k}, 1 / \delta \bar{\gamma}\right) \\
\quad \times \frac{\prod_{l=1, l \neq k}^{u+v} \Gamma\left(\kappa_{8, l}-\kappa_{8, k}\right) \Gamma\left(1+\kappa_{8, k}\right)}{\prod_{l=1}^{r} \Gamma\left(\kappa_{3, l}-\kappa_{8, k}\right)},
\end{aligned}
$$

where $\boldsymbol{\Gamma}(.,$.$) is the upper incomplete Gamma function.$

\section{NumericAl EXAMPLES}

In this section, we present some selected numerical examples to prove the validity of our analytical results and to compare between single FSO link system and mixed RF-FSO relay transmission system. We also show the impact of turbulence conditions and pointing errors on the performance of both systems under both detection techniques (i.e heterodyne and IM/DD). We consider two scenarios of atmospheric turbulence conditions strong (i.e lower values of $\beta_{1}$ and $\beta_{2}$ ) and moderate based on the results reported in [18]. For strong turbulence, we consider the following set of parameters: $\beta_{1}=0.5, \beta_{2}=1.8$, $\alpha_{1}=1.8621, \alpha_{2}=1, \Omega_{1}=1.5074$, and $\Omega_{2}=1$ such that $\lambda$ and $\sigma$ are chosen to satisfy the conditions to be 17 and 9 , respectively. On the other hand, for moderate turbulence conditions, we assume $\alpha_{1}=2.1690, \alpha_{2}=1, \beta_{1}=0.55$, $\beta_{2}=2.35, \Omega_{1}=1.5793$, and $\Omega_{2}=1$ where $\lambda$ and $\sigma$ are chosen to be 28 and 13, respectively [18]. In both cases, it is assumed that $\alpha_{2}$ is an integer to satisfy the condition $\alpha_{2} \lambda$ being an integer.

\section{A. Single Link FSO System}

The outage probability (OP), $P_{\text {out }}$, of a single FSO link under two types of detection techniques experiencing different turbulence condition is presented in Fig. 2. Also, the impact of pointing error is presented in Fig. 3. It is worthy to note that simulation results match the analytical work. In particular, the asymptotic result based on all terms match the analytical perfectly while the rest of the results (based on two or single terms) converge very fast even for low SNR values. We can observe as well that when IM/DD is used, the system

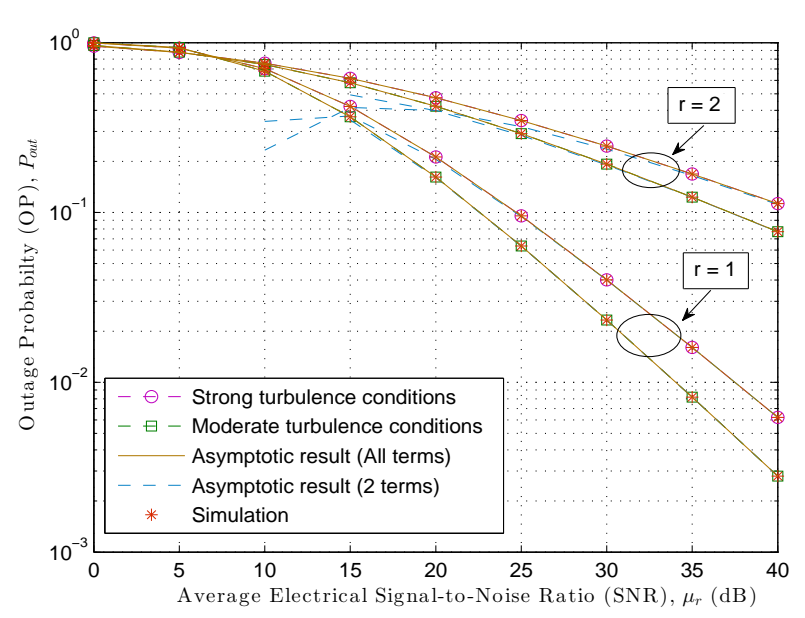

Fig. 2: Outage probability of a single FSO link under strong and moderate turbulence conditions for both detection techniques, heterodyne $(r=1)$ and IM/DD $(r=2)$ with $\xi=1$.

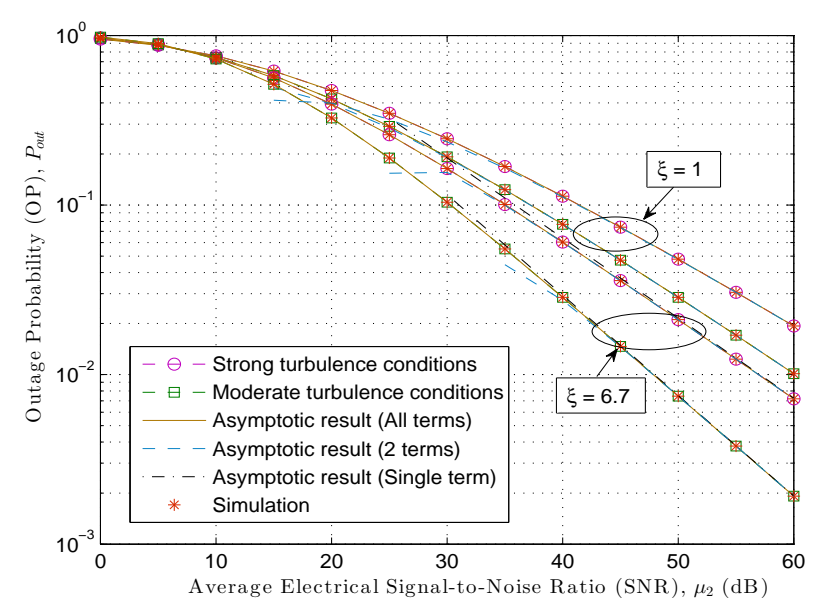

Fig. 3: The impact of pointing errors on the outage probability of a single FSO link under strong and moderate turbulence conditions using IM/DD technique $(r=2)$ with varying pointing errors.

is prone to outage more than if heterodyne is considered. Moreover, high pointing errors effect (i.e $\xi \rightarrow 0$ ) leads to higher probability of system outage.

In addition, the average bit error rate performance under differential binary phase shift keying (DBPSK) modulation, where $p=1$ and $q=1$, for single link FSO system was evaluated in Figs. 4 and 5. We can observe that the simulation results along with the asymptotic (utilizing all terms in the summation as in (25)) are matching with the exact analytical results. However, lower values of $\xi$ (i.e. high pointing errors effect) affect the convergence of asymptotic results (utilizing only the dominant terms explained in II-B2). Specifically, in Fig. 5, we get asymptotic results by utilizing a single dominant term only for the case of $\xi=6.7$. This is mainly due to a significance difference between $\frac{\xi^{2}}{r v}, \frac{\beta_{1}}{r \sigma}, \frac{\beta_{2}}{r \lambda}$ which leads to a dominate term corresponding to $\min \left(\frac{\xi^{2}}{r v}, \frac{\beta_{1}}{r \sigma}, \frac{\beta_{2}}{r \lambda}\right)$. In general, turbulence conditions and pointing errors cause a degradation in the error performance. Yet, heterodyne detection provides better performance than IM/DD. 


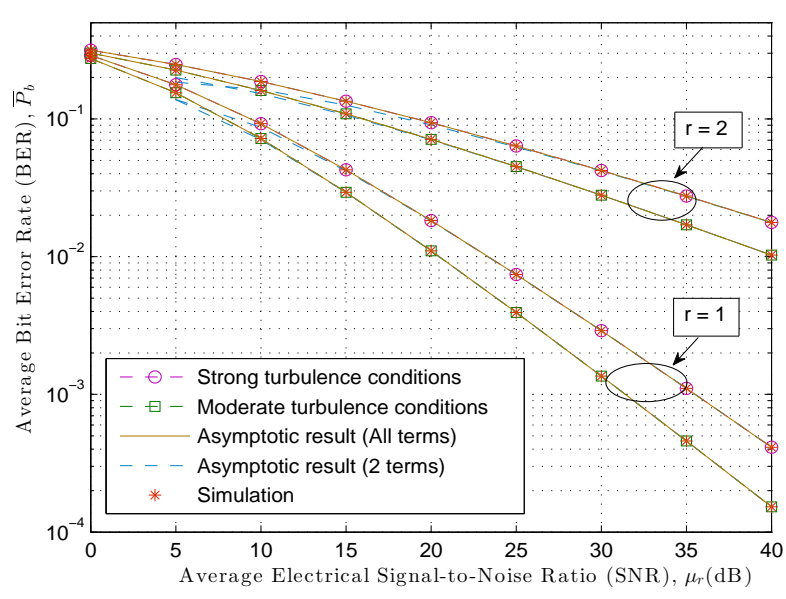

Fig. 4: Average bit error rate of a single FSO link under strong and moderate turbulence conditions for both detection techniques, heterodyne $(r=1)$ and IM/DD $(r=2)$ with $\xi=1$.

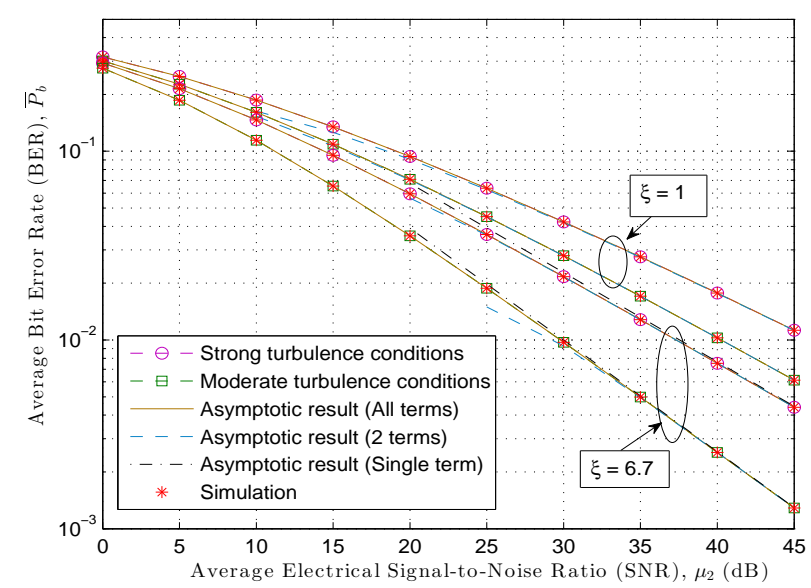

Fig. 5: The impact of pointing errors on the average bit error rate of a single FSO link under strong and moderate turbulence conditions using IM/DD technique $(r=2)$ with varying pointing errors.

Finally, we show the impact of pointing errors on the ergodic capacity of a single FSO link under double GG strong turbulence for IM/DD with varying $\xi$ in Fig. 6. We can notice that high pointing errors effect reduces the capacity of the system. In addition, we have evaluated the asymptotic results via two methods (i.e Meijer's G-function expansion and moments approximation). The asymptotic results derived in (32) (utilizing all terms in the summation) match the exact results perfectly while the other asymptotic (utilizing the dominant terms) converge faster for the case of low pointing errors effect. In the same way, the exact ergodic capacity and asymptotic results for low SNR regime is presented in Fig. 7 as derived earlier in (36).

\section{B. Mixed RF/FSO Dual-hop System}

The outage probability of mixed RF-FSO dual-hop relay transmission system experiencing different turbulence conditions is presented in Figs. 8, 9, and 10 with varying $\xi$ and $r$ to show the impact of pointing errors and the detection

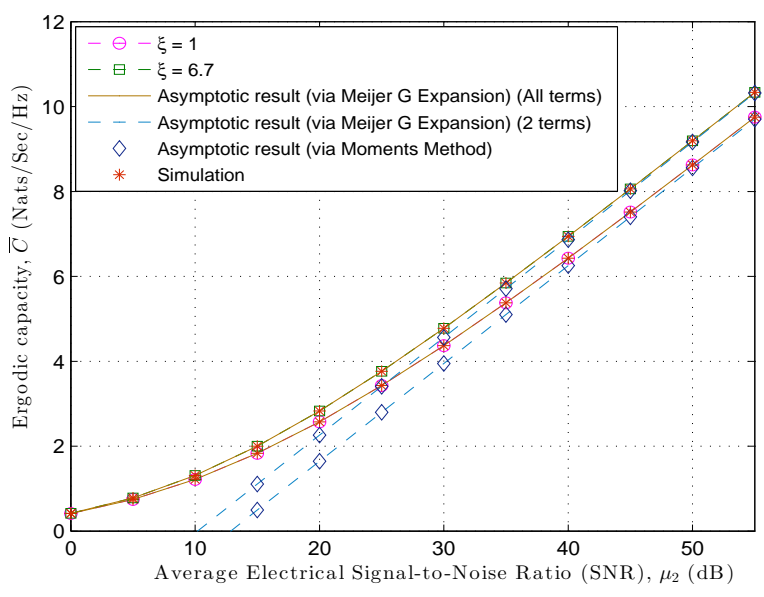

Fig. 6: Ergodic capacity of a single FSO link under strong turbulence conditions for IM/DD $(r=2)$ with varying $\xi$ along with asymptotic results at high SNR.

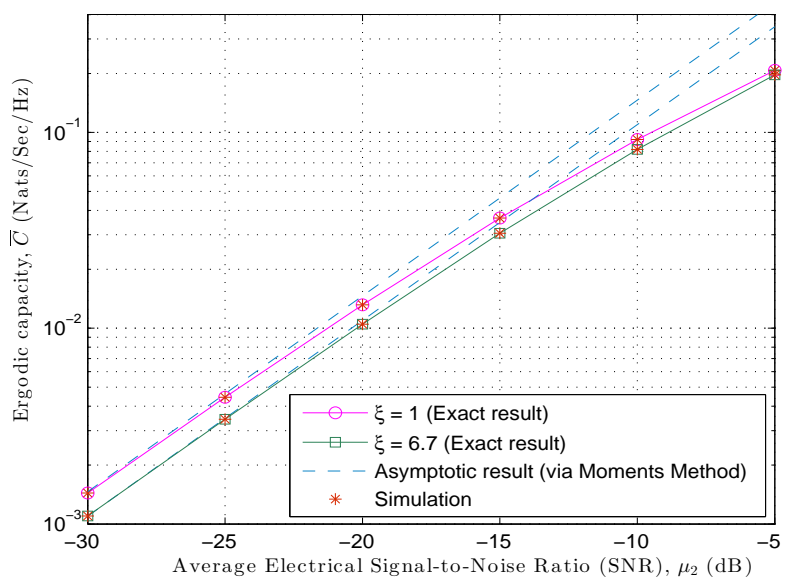

Fig. 7: Ergodic capacity of a single FSO link under strong turbulence conditions for IM/DD $(r=2)$ with varying $\xi$ along with asymptotic results at low SNR.

technique. In particular, in Figs 8 and 9, we fix $\mu_{r, 2}=27 \mathrm{~dB}$ and evaluate the OP with respect to $\bar{\gamma}_{1}$. We can observe that considering heterodyne detection for the FSO link reduces the OP. Moreover, high effect of pointing errors results in higher outage of the system. In Fig. 10, we fix the average SNR of the first link (RF) $\bar{\gamma}_{1}$ to $\in\{20,40\} \mathrm{dB}$ and vary $\mu_{2,2}$ to show system saturation in addition to the asymptotic results using the Meijer's G-function expansion. It is shown that for lower $\bar{\gamma}_{1}$, the system saturates very fast and the asymptotic results converge fast as well.

Similarly, the average bit error rate performance under differential binary phase shift keying (DBPSK) modulation where $p=1$ and $q=1$ for RF-FSO dual hop relay was evaluated in Figs. 11, 12, and 13. More specifically, in Figs. 11 and 12 we evaluate the BER with respect to the first hop SNR $\bar{\gamma}_{1}$. As expected, utilizing heterodyne detection for the FSO link provides better performance than if IM/DD is considered. Also, high pointing errors effect diminish the system performance. On the other hand, in Fig. 13, we evaluate 


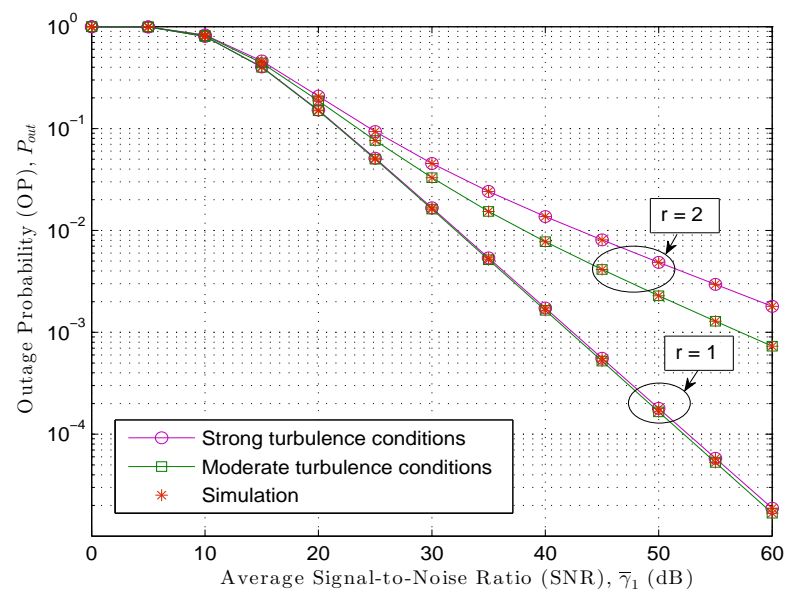

Fig. 8: Outage probability of mixed RF-FSO system link under strong and moderate turbulence conditions for both detection techniques, heterodyne $(r=1)$ and IM/DD $(r=2)$ with $\xi=1$.

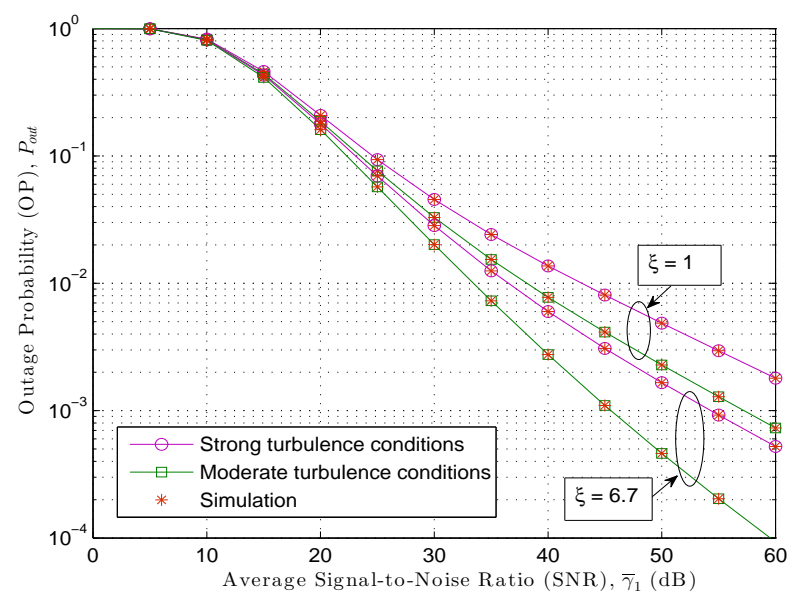

Fig. 9: The impact of pointing errors on the outage probability of mixed FRFSO relay link under strong and moderate turbulence conditions using IM/DD technique $(r=2)$ with varying pointing errors.

the BER with respect to $\mu_{2,2}$ along with asymptotic results. Similar to the OP, low values of $\bar{\gamma}_{1}$ leads to system saturation and better convergence for the asymptotic results (utilizing the dominant terms).

Furthermore, the ergodic capacity of mixed RF-FSO dualhop system operating over double GG turbulence channel with the impact of pointing errors is evaluated in Figs. 14, 15, and 16. Specifically, in Figs. 14 and 15, we evaluate the capacity with respect to $\overline{\gamma_{1}}$. We can notice that heterodyne detection technique provides higher capacity than IM/DD. However, the capacity is still reduced when the pointing errors effect is high. Furthermore, in Fig. 16, we compare the capacity of the system for $\bar{\gamma}_{1}=20 \mathrm{~dB}$ and $\bar{\gamma}_{1}=40 \mathrm{~dB}$. We can observe that higher $\bar{\gamma}_{1}$ yields better system capacity. Finally, in Figs. 14, 15, and 16 , we presented asymptotic results based in all terms as in Eq. (51). Interestingly, it agrees with the analytical results.

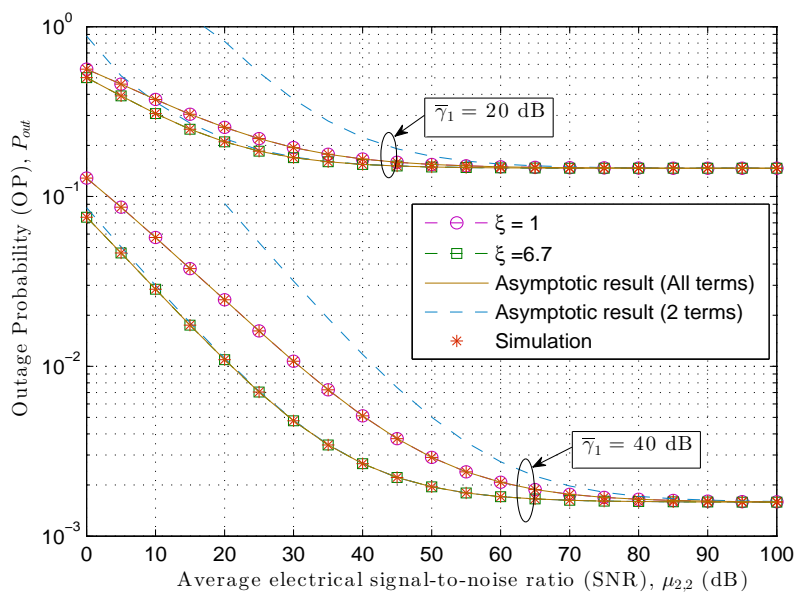

Fig. 10: The impact of $\bar{\gamma}_{1}$ on the outage probability of mixed RF-FSO relay link under strong turbulence conditions using IM/DD technique $(r=2)$ with varying pointing errors.

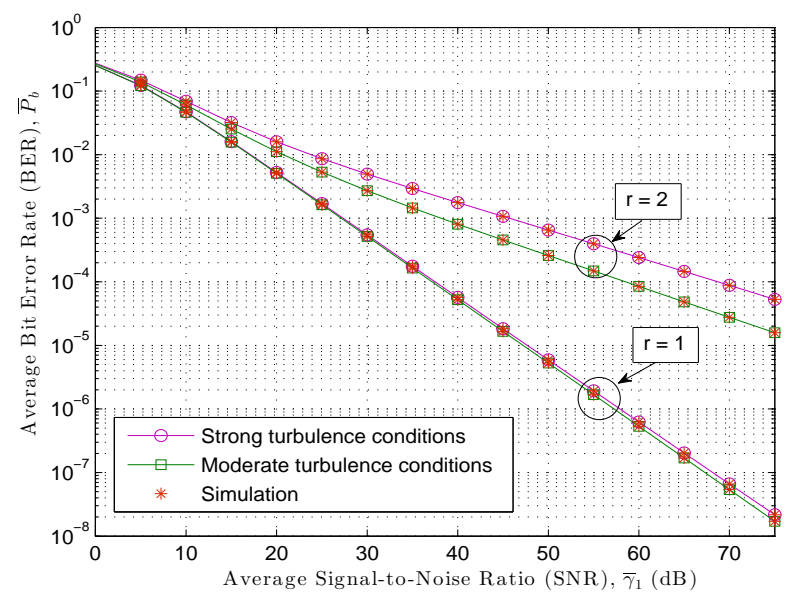

Fig. 11: Average bit error rate of mixed RF-FSO relay link under strong and moderate turbulence conditions for both detection techniques, heterodyne $(r=1)$ and IM/DD $(r=2)$ with $\xi=1$.

\section{CONClusion}

In this paper, we derived the PDF of the irradiance under the impact of pointing errors over double GG turbulence channel. Our PDF matches Kashani et al. results mathematically and numerically when the pointing errors effect approaches zero under IM/DD detection technique. We subsequently derived unified and closed-form expressions for statistical properties of the end-to-end SNR of (i) a single FSO link (ii) mixed RF-FSO relay transmission system taking pointing errors into account. Moreover, we presented link performance analysis for both systems by offering closed-form expressions for the outage probability, the average BER, the higher amount of fading in addition to the ergodic capacity in terms of the Meijer's G-function and bivariate H-function. Finally, for high SNR regime we expressed all of our derivations in terms of elementary functions utilizing an asymptotic expansion for the Meijer's G-function. 


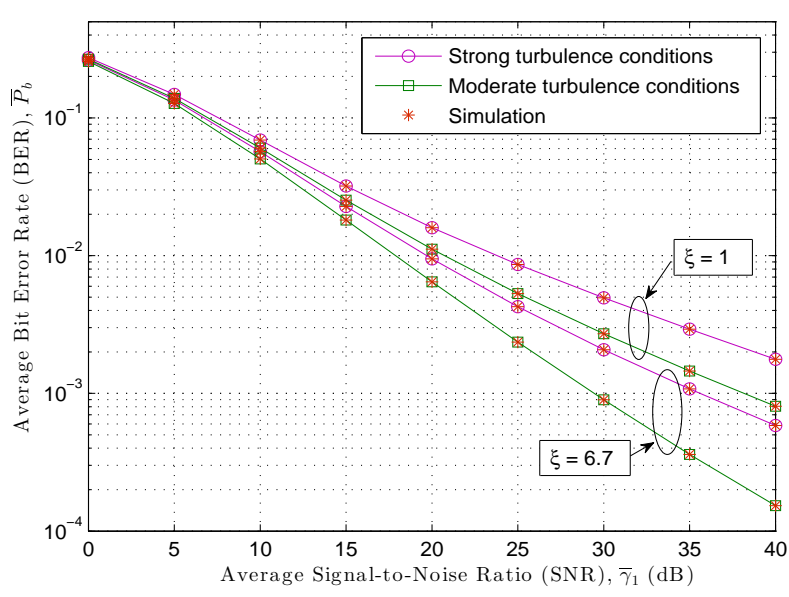

Fig. 12: The impact of pointing errors on the average bit error rate of mixed RF-FSO relay link under strong and moderate turbulence conditions using $\mathrm{IM} / \mathrm{DD}$ technique $(r=2)$ with varying pointing errors.

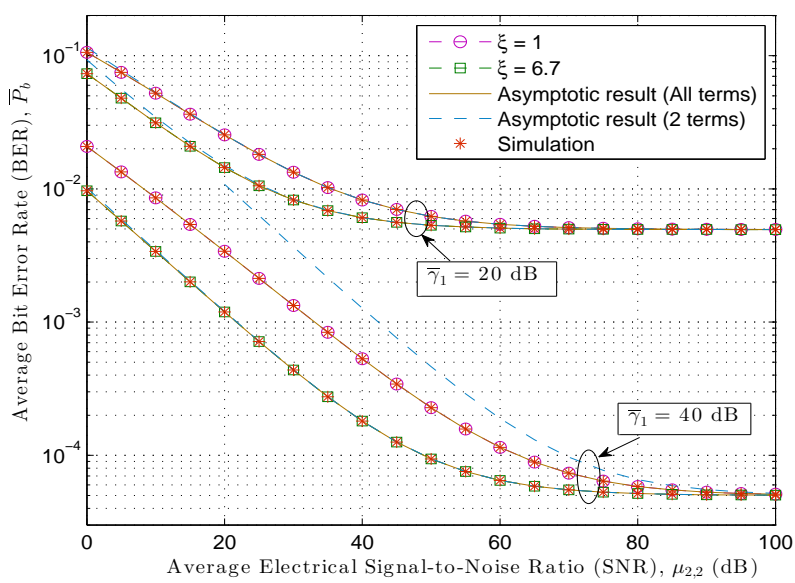

Fig. 13: The impact of $\bar{\gamma}_{1}$ on the average bit error rate of mixed RF-FSO relay link under strong turbulence conditions using IM/DD technique $(r=2)$ with varying pointing errors.

\section{REFERENCES}

[1] H. AlQuwaiee, I. S. Ansari, and M.-S. Alouini, "On the performance of free space optical wireless communication systems over double generalized gamma fading channel," in Proceedings of the $4^{\text {th }}$ International Conference on Communications and Networking (COMNET' 2014), Hammamet, Tunisia, Mar. 2014, pp. 1-5.

[2] D. Kedar and S. Arnon, "Urban optical wireless communication networks: The main challenges and possible solutions," IEEE Communications Magazine, vol. 42, no. 5, pp. S2-S7, May 2004.

[3] S. Arnon, J. Barry, G. Karagiannidis, R. Schober, and M. Uysal, Advanced Optical Wireless Communication Systems. Cambridge University Press, 2012.

[4] L. C. Andrews and R. L. Phillips, Laser Beam Propagation through Random Media. SPIE press, 2005, vol. 152.

[5] W. Gappmair, "Further results on the capacity of free-space optical channels in turbulent atmosphere," IET Communications, vol. 5, no. 9, pp. 1262-1267, Jun. 2011.

[6] H. Sandalidis, T. Tsiftsis, G. Karagiannidis, and M. Uysal, "BER performance of FSO links over strong atmospheric turbulence channels with pointing errors," IEEE Communications Letters, vol. 12, no. 1, pp. 44-46, Jan. 2008

[7] H. Sandalidis, T. Tsiftsis, and G. Karagiannidis, "Optical wireless communications with heterodyne detection over turbulence channels

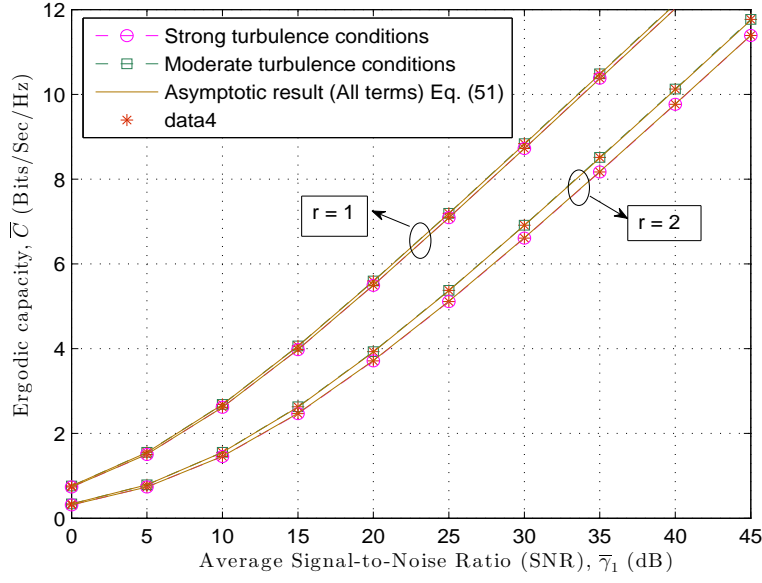

Fig. 14: Ergodic capacity of mixed RF-FSO relay link under strong and moderate turbulence conditions for both detection techniques, heterodyne $(r=1)$ and $\mathrm{IM} / \mathrm{DD}(r=2)$ with $\xi=1$.

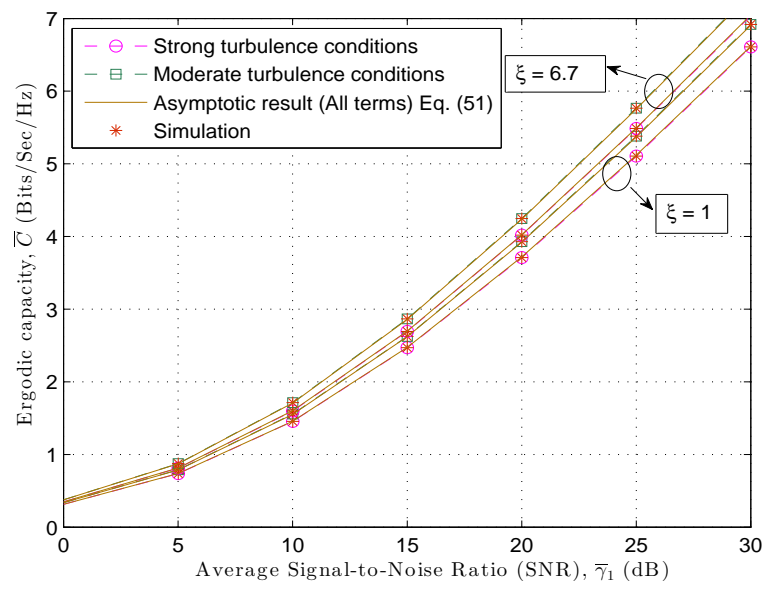

Fig. 15: The impact of pointing errors on the ergodic capacity of mixed RFFSO relay link under strong and moderate turbulence conditions using IM/DD technique $(r=2)$ with varying pointing errors.

with pointing errors," IEEE/OSA Journal of Lightwave Technology, vol. 27 , no. 20 , pp. $4440-4445$, Oct. 2009.

[8] T. Tsiftsis, "Performance of heterodyne wireless optical communication systems over Gamma-Gamma atmospheric turbulence channels," Electronics Letters, vol. 44, no. 5, pp. 372-373, Feb. 2008.

[9] H. Nistazakis, T. Tsiftsis, and G. Tombras, "Performance analysis of free-space optical communication systems over atmospheric turbulence channels," IET Communications, vol. 3, no. 8, pp. 1402-1409, Aug. 2009.

[10] L. Andrews and R. Phillips, "Mathematical genesis of the I-K distribution for random optical fields," Journal of the Optical Society of America A (JOSA A), vol. 3, no. 11, pp. 1912-1919, Nov 1986.

[11] J. H. Churnside and S. F. Clifford, "Log-normal Rician probabilitydensity function of optical scintillations in the turbulent atmosphere," Journal of the Optical Society of America A (JOSA A), vol. 4, no. 10, pp. 1923-1930, Oct. 1987.

[12] M. Al-Habash, L. C. Andrews, and R. L. Phillips, "Mathematical model for the irradiance probability density function of a laser beam propagating through turbulent media," Optical Engineering, vol. 40, no. 8, pp. 1554-1562, Feb. 2001.

[13] I. S. Ansari, F. Yilmaz, and M.-S. Alouini, "A unified performance of free-space optical links over Gamma-Gamma turbulence channels with pointing errors," in Proceedings of IEEE 81st Vehicular Technology Conference (VTC Spring' 2015), Glasgow, Scotland, May 2015.

[14] N. D. Chatzidiamantis, H. G. Sandalidis, G. K. Karagiannidis, S. A. 


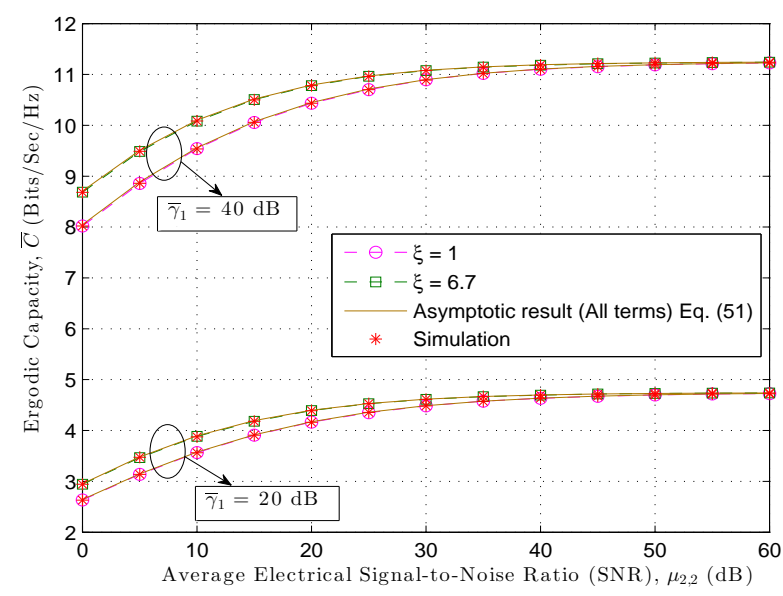

Fig. 16: The impact of $\bar{\gamma}_{1}$ on the ergodic capacity of mixed RF-FSO relay link under strong turbulence conditions using IM/DD technique $(r=2)$ with varying pointing errors.

Kotsopoulos, and M. Matthaiou, "New results on turbulence modeling for free-space optical systems," in Proceedings of IEEE 17th International Conference on Telecommunications (ICT'2010), Doha, Qatar, Apr. 2010, pp. 487-492.

[15] F. Yilmaz and M.-S. Alouini, "A new simple model for composite fading channels: Second order statistics and channel capacity," in Proceedings of the 7th International Symposium on Wireless Communication Systems (ISWCS), York, UK, Sep. 2010, pp. 546-550.

[16] M. Uysal, J. Li, and M. Yu, "Error rate performance analysis of coded free-space optical links over Gamma-Gamma atmospheric turbulence channels," IEEE Transactions on Wireless Communications, vol. 5, no. 6, pp. 1229-1233, Jun. 2006.

[17] I. S. Ansari, F. Yilmaz, and M.-S. Alouini, "Impact of pointing errors on the performance of mixed RF/FSO dual-hop transmission systems," IEEE Wireless Communications Letters, vol. 2, no. 3, pp. 351-354, Jun. 2013.

[18] M. Kashani, M. Uysal, and M. Kavehrad, "A novel statistical model for turbulence-induced fading in free-space optical systems," in Proceedings of the 15th International Conference on Transparent Optical Networks (ICTON), Cartagena, Colombia, June 2013, pp. 1-5.

[19] M. Hasna and M.-S. Alouini, "A performance study of dual-hop transmissions with fixed gain relays," IEEE Transactions on Wireless Communications, vol. 3, no. 6, pp. 1963-1968, Nov. 2004.

[20] F. Yilmaz, O. Kucur, and M.-S. Alouini, "A novel framework on exact average symbol error probabilities of multihop transmission over amplify-and-forward relay fading channels," in Proceedings of the 7th International Symposium on Wireless Communication Systems (ISWCS), York, UK, Sep. 2010, pp. 546-550.

[21] Y. Zhu, Y. Xin, and P.-Y. Kam, "Outage probability of Rician fading relay channels," in Proceedings of Military Communications Conference (MILCOM), Sep. 2006, pp. 1-6.

[22] S. N. Datta, S. Chakrabarti, and R. Roy, "Error analysis of noncoherent FSK with variable gain relaying in dual-hop Nakagami-m relay fading channel," in Proceedings of International Conference on Signal Processing and Communications (SPCOM’2010), Bangalore, India, July 2010, pp. 1-5.

[23] E. Lee, J. Park, D. Han, and G. Yoon, "Performance analysis of the asymmetric dual-hop relay transmission with mixed RF/FSO links," IEEE Photonics Technology Letters, vol. 23, no. 21, pp. 1642-1644, Nov. 2011.

[24] A. Farid and S. Hranilovic, "Outage capacity optimization for free-space optical links with pointing errors," Journal of Lightwave Technology, vol. 25 , no. 7, pp. 1702-1710, July 2007.

[25] H. Samimi and M. Uysal, "End-to-end performance of mixed RF/FSOtransmission systems," J. Opt. Commun. Netw., vol. 5, no. 11, pp. 1139-1144, Nov 2013.

[26] E. Stacy, "A generalization of the Gamma distribution," The Annals of Mathematical Statistics, vol. 33, no. 3, pp. 1187-1192, Sep. 1962.

[27] I. S. Gradshteyn and I. M. Ryzhik, "Table of Integrals, Series and Products," Academic Press, New York, 2000.
[28] F. Yilmaz and M.-S. Alouini, "A novel unified expression for the capacity and bit error probability of wireless communication systems over generalized fading channels," IEEE Transactions on Communications, vol. 60, no. 7, pp. 1862-1876, 2012.

[29] A. Jurado-Navas, J. Garrido-Balsells, J. Paris, and A. Puerta-Notario, "Numerical simulations of physical and engineering processes," 2011.

[30] I. Wolfram, Mathematica Edition: Version 8.0. Champaign, Illinois: Wolfram Research Inc., 2010.

[31] T. Tsiftsis, H. Sandalidis, G. Karagiannidis, and M. Uysal, "Optical wireless links with spatial diversity over strong atmospheric turbulence channels," IEEE Transactions on Communications, vol. 8, no. 2, pp. 951-957, Feb. 2009.

[32] M. Niu, J. Cheng, and J. Holzman, "Error rate performance comparison of coherent and subcarrier intensity modulated optical wireless communications," IEEE/OSA Journal of Optical Communications and Networking, vol. 5, no. 6, pp. 554-564, June 2013.

[33] I. S. Ansari, F. Yilmaz, and M.-S. Alouini, "On the performance of mixed RF/FSO variable gain dual-hop transmission systems with pointing errors," in Proceedings of IEEE 78th Vehicular Technology Conference (VTC Fall '13), Las Vegas, USA, Sep. 2013.

[34] F. Yilmaz and M.-S. Alouini, "Novel asymptotic results on the highorder statistics of the channel capacity over generalized fading channels," in Proceedings of IEEE 13th International Workshop on Signal Processing Advances in Wireless Communications (SPAWC' 2012), Cesme, Turkey, Jun. 2012, pp. 389-393.

[35] I. S. Ansari, S. Al-Ahmadi, F. Yilmaz, M.-S. Alouini, and H. Yanikomeroglu, "A new formula for the BER of binary modulations with dual-branch selection over generalized- $K$ composite fading channels," IEEE Transactions on Communications, vol. 59, no. 10, pp. 2654-2658, Oct. 2011.

[36] Z. Wang and G. Giannakis, "A simple and general parameterization quantifying performance in fading channels," IEEE Transactions on Communications, vol. 51, no. 8, pp. 1389-1398, Aug 2003.

[37] A. Lapidoth, S. Moser, and M. Wigger, "On the capacity of free-space optical intensity channels," IEEE Transactions on Information Theory, vol. 55, no. 10, pp. 4449-4461, Oct 2009.

[38] S. Arnon, J. Barry, G. Karagiannidis, R. Schober, and M. Uysal, Advanced Optical Wireless Communication Systems. Cambridge University Press, 2012.

[39] M. K. Simon and M.-S. Alouini, Digital Communication over Fading channels. New York: Wiley, 2000.

[40] A. Annamalai, R. Palat, and J. Matyjas, "Estimating ergodic capacity of cooperative analog relaying under different adaptive source transmission techniques," in Proceedings of 2010 IEEE Sarnoff Symposium, Princeton, NJ, USA, Apr. 2010, pp. 1-5.

[41] A. Mathai, R. K. Saxena, and H. J. Haubold, The H-function: Theory and Applications. Springer, 2010.

[42] P. Mittal and K. Gupta, "An integral involving generalized function of two variables," Proceedings of the Indian Academy of Sciences Section A, vol. 75, no. 3, pp. 117-123, Mar. 1972.

[43] K. Peppas, "A new formula for the average bit error probability of dualhop amplify-and-forward relaying systems over generalized shadowed fading channels," IEEE Wireless Communications Letters, vol. 1, no. 2, pp. 85-88, April 2012.

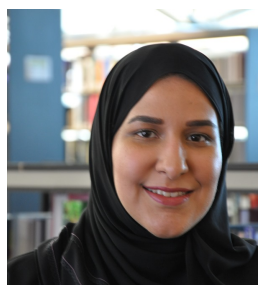

Hessa AlQuwaiee (S'09) received the B.S degree in Computer Engineering from Prince Mohamed University (PMU), Alkhobar, Saudi Arabia in 2011. In 2008, she was awarded the discovery scholarship to complete graduate studies in King Abdullah University of Science and Technology (KAUST). In 2013, she earned her M.S. degree in Electrical Engineering. She is now toward her PhD degree at KAUST. Her current research interests include, but not limited to, channel characterization and performance analysis of optical wireless communications, body-to-body communications, diversity combining techniques and MIMO systems. 
Imran Shafique Ansari was born in India in 1987. He completed his B.S. in Computer Engineering from King Fahd University of Petroleum and Minerals (KFUPM) in 2009 (with First Honors) and M.S. from King Abdullah University of Science and Technology (KAUST) in 2010. Currently, he is a $\mathrm{PhD}$ Candidate at KAUST. From May 2009 through Aug. 2009, he was a visiting scholar with Michigan State University (MSU) and from Jun. 2010 through Aug. 2010, he was a research intern with Carleton University. Imran has been a student member of IEEE and IET since 2007 and has served in various capacities. He has served on IET CC-EMEA (Communities Committee-Europe, Middle-East and Africa) for a complete term from Oct. 2010-Sep. 2013 and has been re-elected to serve for another term from Oct. 2015-Sep. 2018. He is an active reviewer for various IEEE transactions and various other journals. He has served as a TPC for various IEEE conferences. He is a recipient of appreciation for an exemplary reviewer for IEEE Wireless Communications Letters (WCL) 2014, a recipient of post-doctoral research award (PDRA) (first cycle) with Qatar national research foundation (QNRF) 2014, a recipient of KAUST academic excellence award (AEA) 2014, and a recipient of IEEE Richard E. Merwin student scholarship award Jul. 2013. Imran has authored/co-authored around 25 journal and conference publications. His current research interests include free-space optics (FSO), channel modeling/signal propagation issues, relay/multihop communications, and diversity reception techniques among others.

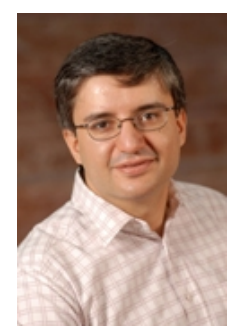

Mohamed-Slim Alouini (S'94, M'98, SM'03, F'09) was born in Tunis, Tunisia. He received the Ph.D. degree in Electrical Engineering from the California Institute of Technology (Caltech), Pasadena, CA, USA, in 1998. He served as a faculty member in the University of Minnesota, Minneapolis, MN, USA, then in the Texas A\&M University at Qatar, Education City, Doha, Qatar before joining King Abdullah University of Science and Technology (KAUST), Thuwal, Makkah Province, Saudi Arabia as a Professor of Electrical Engineering in 2009. His current research interests include the modeling, design, and performance analysis of wireless communication systems. 